\title{
Theory of Monomer-Dimer Systems ${ }^{\star}$
}

\author{
Ole J. HeilmanN ${ }^{\star \star}$ and Elliott H. Lieb \\ Department of Mathematics \\ Massachusetts Institute of Technology, Cambridge, Massachusetts, USA
}

Received November 30, 1971

\begin{abstract}
We investigate the general monomer-dimer partition function, $P(x)$, which is a polynomial in the monomer activity, $x$, with coefficients depending on the dimer activities. Our main result is that $P(x)$ has its zeros on the imaginary axis when the dimer activities are nonnegative. Therefore, no monomer-dimer system can have a phase transition as a function of monomer density except, possibly, when the monomer density is minimal (i.e. $x=0$ ). Elaborating on this theme we prove the existence and analyticity of correlation functions (away from $x=0$ ) in the thermodynamic limit. Among other things we obtain bounds on the compressibility and derive a new variable in which to make an expansion of the free energy that converges down to the minimal monomer density. We also relate the monomer-dimer problem to the Heisenberg and Ising models of a magnet and derive Christoffell-Darboux formulas for the monomer-dimer and Ising model partition functions. This casts the Ising model in a new light and provides an alternative proof of the Lee-Yang circle theorem. We also derive joint complex analyticity domains in the monomer and dimer activities. Our considerations are independent of geometry and hence are valid for any dimensionality.
\end{abstract}

\section{Introduction}

A monomer-dimer system is specified by a graph, $G$ (also called a lattice in the physics literature), together with a family of weights (or Boltzmann factors) assigned to the edges of $G$. The precise definition of a weighted graph is given in Section II, but for the present we shall assume the reader is familiar with the concept. Dimers can be placed on the edges of $G$ so that no vertex has more than one dimer. Uncovered vertices are called monomers and have a fugacity which we call $x$. One can also define related problems, such as the monomer-trimer problem, and although the history of these various problems are intertwined we shall consider only the monomer-dimer problem in this paper.

We shall answer the question whether, as the monomer concentration is varied, a phase transition can occur for an infinite system. Our answer,

* Work supported by National Science Foundation Grant GP-26526.

$\star \star$ Permanent address: Kemisk Laboratorium III, the H.C. Ørsted Institute, University of Copenhagen, 2100 Copenhagen, Denmark. 
which is independent of the geometry of the graph, is that the only place a singularity can possibly occur is at minimum monomer density (which is zero for lattices normally considered).

Although this paper is mathematical in nature and is not a survey paper, it might be useful to begin with a brief historical sketch (with no pretense of completeness, and therefore with apologies to the authors we have unintentionally omitted) to show how monomer-dimer systems are related to chemistry and physics.

The problem of placing nonoverlapping dimers on a lattice goes back at least to 1935 when Roberts [1] considered the problem of absorption of oxygen and hydrogen on a tungsten surface. His assumptions were based on kinetic considerations: If oxygen molecules are absorbed on the surface in a single layer with the two oxygen atoms of a molecule covering two neighboring tungsten atoms, how does the chance of finding two neighboring tungsten atoms both unoccupied depend on the density of absorbed oxygen and what is the average maximum density? Roberts attacked the problem by a straightforward Monte Carlo calculation [1-3] and later theoretically by applying the Bethe approximation [4]. More extensive Monte Carlo calculations were undertaken after the appearance of high speed computers $[5,6]$, and the problem has been given rigorous mathematical treatment in one dimension by McQuistan [7-10]. It should be noted that since it is assumed that molecules once absorbed do not move or leave the surface, then the statistics of this problem differs from the statistics of the ordinary, equilibrium monomerdimer problem treated in the present paper and we shall deal no further with it. It is a pecularity of the Bethe approximation that the two problems are equivalent in first order.

The earliest treatment of the equilibrium monomer-dimer problem is due to Fowler and Rushbrooke [11], who took up the problem to settle a question raised earlier in 1937 by Guggenheim [12], namely whether deviations in the properties of a binary mixture from those of an ideal mixture could be caused solely by a difference in size of the two components. The Fowler-Rushbrooke paper discusses approximations valid at low dimer density and treats the pure dimer covering (i.e., no monomers) problem by finding eigenvalues for the transfer matrix for narrow strips.

The monomer-dimer problem was soon attacked in the Bethe approximation or random mixing approximation [4,13-17], in which cases it is easy to include an interaction between neighboring vertices both of which are occupied by dimers. Almost simultaneously, the more general problem of monomer-polymer mixtures was attacked in the same approximations $[18,19,21,22]$ and in the slightly cruder approximations of the Flory-Huggins theory [23]. A description of these methods 
and the results can be found in Guggenheim's book on mixtures [24]. Rushbrooke et al. [25] attacked the monomer-polymer problem by series expansions valid at low polymer density.

Newer and more accurate results on the monomer-dimer problem have been obtained by series expansions valid at low dimer density [26-29] (the results of our paper show that the choice of expansion parameter in [27] actually makes the expansion valid at all dimer densities which are lower than the density of the pure dimer covering; for a proof of this fact see Chapter IX). Other results have been obtained by finding the largest eigenvalue of the transfer matrix either numerically for narrow strips [30] or by a variational procedure [31]. A numerical comparison of the entropy of mixing as calculated from the different approximations can be found in [32]. Monte Carlo calculations have also been performed [33].

The application of monomer-dimer systems as models for real, physical systems is rather limited. Fair agreement has been obtained for binary mixtures like benzene-diphenyl [34-36], benzene-diphenylmethane [35] and benzene-dibenzyl [35]. Mixtures like hexane-cetane [37], do not fit the model because of the flexibility of the "monomer" (hexane) [38]. Other possibilities are mixtures of monovalent and divalent ions; two examples are absorption of sodium and cupri-ions on a sterate film on water [13] and melted $\mathrm{Li}_{2} \mathrm{O}-\mathrm{NiO}$ mixtures [39]. The monomerdimer theory has also found application in the cell-cluster theory [40] and in improvements of the Debye-Hückel theory when some of the ions are large [41].

The first rigorous results on the monomer-dimer problem were mostly of a negative nature $[42,43]$, and the earliest breakthrough came in the related dimer-covering problem (i.e., zero monomer density). The theory of this problem received a great impetus when the exact solution for planar lattices was discovered by Kasteleyn, Temperley and Fisher in 1961 [44-46]. We shall only mention the dimer-covering problem briefly in this paper, primarily because the nature of the pure dimer problem is dependent on the geometry of the lattice, while our main concern is with results independent of the structure of the lattice; part of the succeeding development of the dimer covering problem can be found in references [47-53].

The solution of the planar dimer-covering problem made it possible to calculate the monomer-monomer correlation function in the case of only two monomers $[54,55]$. Other rigorous results on the monomerdimer problems are lower bounds on the free energy obtained by Bondy and Welsh [56] and Hammersley and Menon [57, 58], and Hammersley's proof [59] of the existence of the thermodynamic limit in the sense of Van Hove for simple cubic lattices of arbitrary dimension. 
Apart from the numerical computation of the monomer-dimer partition function for specific simple lattices, the central theoretical question to be answered is this: can a monomer-dimer system have a phase transition? The numerical work previously cited had led to the conjecture that a monomer-dimer phase transition did not occur on simple lattices but a proof was lacking. (It is worth pointing out that similar conclusions were also drawn about monomer-trimer systems, but we now have an example of a special monomer-trimer system that can be proved to have a phase transition [60].) It is the purpose of this paper to prove that a phase transition cannot occur for any lattice and, since our results are quite general, no mention is made of lattice geometry except insofar as it is necessary to prove the existence of the thermodynamic limit (Section VIII). (Our method of proof is different from Hammersley's [59].) In Section II we give the basic definitions and in Section III evaluate the monomer-dimer partition function for some simple lattices by way of illustration. In Section IV we give our main results on the zeros of the monomer-dimer partition function, when considered as a polynomial in $x$, for positive edge weights. The zeros lie on the imaginary axis. This result can be generalized to non-positive (complex) edge weights (Theorem 4.9). One practical consequence of locating the zeros is that by changing the variable from $x$ to some simple function of $x$ one can make a Taylor expansion of the free energy for all non-zero monomer densities and hence can undertake numerical calculations with greater confidence than before, including bounds on the error. This is shown in Section IX.

Section $\mathrm{V}$ relates the monomer-dimer problem to various magnetic systems and Section VI gives Christoffel-Darboux formulas for the monomer-dimer and magnetic system partition functions. In Section VII we use our knowledge of the location of the zeros to give bounds on the compressibility and show that these are stronger than the bounds derived using Ginibre's general method. In Section IX we prove the existence and analyticity of correlation functions and of the free energy.

A preliminary report of this work was given in Heilmann and Lieb [61]. Shortly thereafter Kunz [62] and Gruber and Kunz [63], in their work on the general monomer-polymer problem, announced several of the same theorems, notably the one on the location of the zeros.

To conclude this introduction we wish to draw attention to the fact that our monomer-dimer result is related to another problem in statistical mechanics - the nearest neighbor exclusion (or hard core lattice gas) model. Suppose that $G$ is an unweighted graph on whose vertices particles may be placed provided no edge has particles at both of its terminal vertices. We might expect a phase transition to occur as the particle density is varied. Indeed, Dobrushin [64] has shown this to be 
the case for the square and cubic lattice and Heilmann [60] has extended this conclusion to the planar triangular and face centered cubic lattices. If, however, $G$ is the line graph of a graph $G^{*}$ (i.e., the vertices of $G$ are the edges of $G^{*}$ and two vertices of $G$ are connected if the corresponding edges of $G^{*}$ have a common vertex) then the exclusion problem on $G$ is the same as the monomer-dimer problem (with unit edge weights) on $G^{*}$. Hence the exclusion problem on $G$ has no phase transition. An example of this is the Kagome lattice which is the line graph of the planar hexagonal lattice.

\section{Basic Definitions}

The notation and terminology used in this paper will differ in certain respects from those employed in the previous paper [61], partly to make the notation more convenient and partly to bring the terminology into closer agreement with what is generally accepted.

Graphs. The basic terminology for unweighted graphs will be that suggested by Essam and Fisher [65] and we shall not repeat their definitions. Unfortunately, they did not suggest any terminology for weighted graphs: If $G$ is a graph then we shall associate a complex number, $W(i, j)$, called an edge weight, with each unordered pair of vertices, $[i, j]$, in $G$. Unless otherwise stated, $W(i, j)$ will be assumed non-negative for all pairs, $[i, j]$, and positive if there is an edge connecting $i$ and $j$. We shall further associate a complex number, $x_{i}$ (called a vertex weight), with each vertex in $G$. We shall use $N(G)$ (or just $N$ if no ambiguity is caused) for the number of vertices in $G$. We shall write $G-G^{\prime}$ for the section graph of $G$ which is obtained by deleting the vertices in the subgraph, $G^{\prime}$. If $G^{\prime}$ consists only of the $i^{\prime}$ th vertex we shall write $G-i$.

Definition 2.1. A graph is said to be Hamiltonian if it contains a Hamilton walk. i.e. if it is possible to number the vertices in the graph such that

$$
W(1,2) W(2,3) \ldots W(N-1, N) \neq 0 .
$$

Dimers. A dimer is a molecule which can be placed on the graph, $G$, such that it covers an edge and the two vertices on which the edge is incident. We shall use $\langle i, j\rangle$ for the dimer which covers vertices $i$ and $j$. A dimer arrangement, $D$, is a set of dimers placed on $G$ such that no vertex is covered by more than one dimer. The set of vertices of $G$ covered by the dimers in $D$ will be denoted by $[D]$. The set of all dimer arrangements will be denoted by $\mathscr{D}$. A dimer arrangement which covers all vertices in $G$ is called a dimer covering.

Canonical Weights. For a dimer arrangement, $D$, the canonical weight, $W(D)$, is given by

$$
W(D)=\prod_{\langle i, j\rangle \in D} W(i, j) .
$$


The d-dimer partition function (or weight) of all dimers arrangements with $d$ dimers is

$$
Z_{d}=\sum_{\substack{D \in \mathscr{O} \\ \# D=d}} W(D)
$$

where $\# D$ is the number of dimers in $D$.

If we denote the largest integer in $N / 2$ by $M$, i.e.

$$
M=[N / 2] \text {, }
$$

then we can define the generating function for $Z_{d}$ :

$$
P(G ; x)=\sum_{d=0}^{M} Z_{d} x^{N-2 d} .
$$

Obviously $P(G ; x)$ is a polynomial in $x$ of degree $N$ and will be called simply the partition function (of the weighted graph $G$ ). It will prove convenient to define $P(G ; x)$ for a graph having no vertices by

$$
P(\emptyset ; x)=1 \text {. }
$$

Remark. If $W(i, j)$ is written as

$$
W(i, j)=\exp \left(-\beta J_{i j}\right),
$$

then $Z_{d}$ can be interpreted as the canonical partition function for $d$ dimers on the graph, $G$, if $J_{i j}$ is the energy gained by placing a dimer on the two vertices $i$ and $j$. Furthermore, if $x$ is taken to be the activity of a monomer, and if it is supposed that all vertices not covered by a dimer contain a monomer, then $P(G ; x)$ is the grand canonical partition function for the distribution of monomers and dimers on $G$. Alternatively, $P(G ; 1)$ can be considered to be the grand canonical partition function for the distribution of hard dimers on $G$; this analogy is particularly useful since much of the standard theory of statistical mechanics applies directly to this case.

Further polynomials: Besides $P(G ; x)$ we shall also need two related polynomials, $Q(G ; x)$ and $R(G ; x)$ :

$$
\begin{aligned}
& Q(G ; x)=i^{-N} P(G ; i x), \\
& R(G ; x)= \begin{cases}P(G ; \sqrt{x}), & N \text { even, } \\
P(G ; \sqrt{x}) / \sqrt{x}, & N \text { odd } .\end{cases}
\end{aligned}
$$

A Generating Function in $N$ Variables. Finally we shall need a more general form of $P(G ; x)$ which includes the vertex weights:

$$
P\left(G ; x_{1}, x_{2}, \ldots, x_{N}\right)=\sum_{D \in \mathscr{Q}} W(D) \prod_{i \in G-[D]} x_{i},
$$


where the sum runs over all dimer arrangements on $G$ and the product runs over all vertices of $G$ which are not in $[D]$.

One can easily derive the following relation:

$$
P\left(G ; m_{1} x, m_{2} x, \ldots, m_{N} x\right)=\left(\prod_{i=1}^{N} m_{i}\right) P\left(G^{\prime} ; x\right),
$$

where the graph $G^{\prime}$ differs from $G$ only in the edge weights which are related by:

$$
m_{i} m_{j} W^{\prime}(i, j)=W(i, j) .
$$

In particular one has

$$
P(G ; x, x, \ldots, x)=P(G ; x) .
$$

An important property of $P\left(G ; x_{1}, \ldots, x_{N}\right)$ is that it is linear in each variable, i.e. considered as a function of $x_{j}$ it is a polynomial of degree one.

\section{Examples of Partition Functions}

In this section we shall give closed form expressions for $Q(G, x)$ for some simple graphs. Most of these results are well known and, as the purpose of this section is primarily that of illustration, it may be omitted without any loss of continuity.

Example 1. The Linear Chain. In this case the weights are given by

$$
\begin{aligned}
W(i, j) & =1, & & \text { if } \quad|i-j|=1 \\
& =0, & & \text { otherwise } .
\end{aligned}
$$

If we define $L(N)$ to be the linear chain with $N$ vertices and edge weights as given by (3.1), one has the following recursion formula for $N \geqq 2$ :

$$
Q(L(N) ; x)=x Q(L(N-1) ; x)-Q(L(N-2) ; x),
$$

where the first term of the right hand side corresponds to a monomer at the $N^{\prime}$ th vertex and the second term corresponds to a dimer covering the vertices $N$ and $N-1$. Since one has

$$
Q(L(0) ; x)=1, \quad Q(L(1) ; x)=x,
$$

one easily find that

$$
Q(L(N) ; x)=U_{N}\left(\frac{1}{2} x\right),
$$

where $U_{n}(x)$ is the Chebyshev polynomial of the second kind of degree $n$ which is defined by

$$
\begin{aligned}
U_{n}(x) & =\sin ((n+1) \theta) / \sin \theta, \\
\cos \theta & =x .
\end{aligned}
$$


It is easily seen that the zeros of $Q(L(N) ; x)$ are real and lie in the interval $|x|<2$. Consequently the zeros of $P(L(N) ; x)$ are pure imaginary and are limited by $|\operatorname{Im}(x)|<2$. It should be noted that this bound on the roots is the best possible bound that is independent of $N$.

Example 2. The Polygon. In this case the weights are given by

$$
W(i, j)=\left\{\begin{array}{ll}
1 & \text { if }|i-j|=1 \\
0 & \text { otherwise }
\end{array} \text { or }(i, j)=(1, N),\right.
$$

If we use $P(N)$ for the polygon with $N$ vertices and all edge weights equal to one we have the following formula for $N \geqq 2$ :

$$
Q(P(N) ; x)=x Q(L(N-1) ; x)-2 Q(L(N-2) ; x),
$$

where the first term of the right hand side corresponds to a monomer on the $N^{\prime}$ th vertex and the second term corresponds to a dimer which includes the $N$ 'th vertex (and which can be either $\langle N-1, N\rangle$ or $\langle 1, N\rangle$ ). Using Eq. (3.4) one finds

$$
Q(P(N) ; x)=2 T_{N}\left(\frac{x}{2}\right),
$$

where $T_{n}(x)$ is the Chebyshev polynomial of the first kind of degree $n$, defined by:

$$
\begin{aligned}
& T_{n}(x)=\cos (n \theta), \\
& \cos \theta=x .
\end{aligned}
$$

It is easily seen that the zeros have the same properties and the same bounds as those given for the linear chain.

Example 3. The Complete Graph. In this case the edge weights are given by

$$
W(i, j)=1, \quad \text { all }(i, j) .
$$

If we use $K(N)$ for the complete graph with $N$ vertices and edge weights equal to one we have the following recursion formula for $N \geqq 2$ :

$$
Q(K(N) ; x)=x Q(K(N-1) ; x)-(N-1) Q(K(N-2) ; x),
$$

where again the first term of the right hand side corresponds to a monomer on the $N$ 'th vertex and the second term corresponds to a dimer including the $N$ 'th vertex. Since one has

it follows that

$$
Q(K(0) ; x)=1, \quad Q(K(1) ; x)=x,
$$

$$
Q(K(N) ; x)=H e_{N}(x),
$$

where $H e_{n}(x)$ is the Hermite polynomial of degree $n$ defined by

$$
H e_{n}(x)=(-1)^{n} e^{x^{2} / 2} \frac{d^{n}}{d x^{n}} e^{-x^{2} / 2} .
$$


If one renormalizes the edge weights to

$$
W^{\prime}(i, j)=(N-1)^{-1}, \quad(N \geqq 2),
$$

then one gets, with $K^{\prime}(N)$ for the complete graph with edge weights normalized according to (3.16):

$$
Q\left(K^{\prime}(N) ; x\right)=(N-1)^{-N / 2} H e_{N}(x \sqrt{N-1}) .
$$

As before one finds that the zeros of $Q\left(K^{\prime}(N) ; x\right)$ are real and the best $N$ independent bound is $|x|<2$ [66].

Example 4. The Bethe Graph. We shall define the rooted Bethe graph of degree $d$ and order $n, B(d, n)$, as follows: $B(d, 1)$ consists of a single vertex called the root; $B(d, n)$ consists of a vertex called the root, which is connected by edges to the roots of $d-1$ graphs $B(d, n-1)$. With edge weights equal to one on all edges, one finds in the usual manner for $n \geqq 2$ that

$$
\begin{aligned}
Q(B(d, n) ; x)= & x[Q(B(d, n-1) ; x)]^{d-1} \\
& -(d-1)[Q(B(d, n-2) ; x)]^{d-1}[Q(B(d, n-1) ; x)]^{d-2} .
\end{aligned}
$$

Defining the quantity $\hat{Q}_{n}(x)$ for $n \geqq 2$ by

$$
\hat{Q}_{n}(x)=Q(B(d, n) ; x)\left[\prod_{i=1}^{n-1} Q(B(d, i) ; x)\right]^{2-d},
$$

one gets for $n \geqq 3$

with

$$
\begin{aligned}
& \hat{Q}_{n}(x)=x \hat{Q}_{n-1}(x)-(d-1) \hat{Q}_{n-2}(x), \\
& \hat{Q}_{1}(x)=x, \quad \hat{Q}_{2}(x)=x^{2}-(d-1) .
\end{aligned}
$$

Therefore, $\hat{Q}_{n}(x)$ is a polynomial and, by comparison with (3.2) and (3.4),

$$
\hat{Q}_{n}(x)=(d-1)^{n / 2} U_{n}\left(\frac{1}{2} x(d-1)^{-\frac{1}{2}}\right),
$$

where $U_{n}(x)$ is again the Chebyshev polynomial defined in Eq. (3.5). The inversion of (3.19) is given by

$$
Q(B(d, n) ; x)=\hat{Q}_{n}(x) \prod_{j=1}^{n-1}\left[\hat{Q}_{j}(x)\right]^{(d-2)(d-1)^{(n-1-j)}},
$$

for $n>1$, while

$$
Q(B(d, 1) ; x)=\hat{Q}_{1}(x)=x .
$$

The ( $n$ independent) bound on the zeros of $Q(B(d, n) ; x)$ is seen to be $|x|<2 \sqrt{d-1}$. 


\section{Location of Zeros of Monomer Dimer Partition Functions}

All the proofs in this section will be based on the two-step recurrence relation satisfied by the monomer-dimer polynomials. For $P(G ; x)$ it reads (with $i$ being any vertex in $G$ ):

$$
P(G ; x)=x P(G-i ; x)+\sum_{j \in G-i} W(i, j) P(G-i-j ; x) .
$$

The same is true for $Q(G ; x)$ if the plus sign is replaced by a minus sign. The first term on the right is the contribution of a monomer placed on the $i$ 'th vertex, while the summation over $j$ gives the contribution of all the ways of placing a dimer at $i$. Obviously, if $x$ is a zero of $P(G ; x)$ then $i x$ is a zero of $Q(G ; x)$ and conversely.

Lemma 4.1. If $G$ is a complete graph (i.e. $W(i, j)>0$ for all pairs $[i, j])$ then the zeros of $Q(G ; x)$ are all real. Furthermore, if $i$ is any vertex in $G$ then the zeros of $Q(G ; x), a_{1}, a_{2}, \ldots, a_{N}$, and the zeros of $Q(G-i, x)$, $a_{1}^{\prime}, a_{2}^{\prime}, \ldots, a_{N-1}^{\prime}$ obey a strict interlacing relation:

$$
a_{1}<a_{1}^{\prime}<a_{2}<a_{2}^{\prime}<\cdots<a_{N-1}^{\prime}<a_{N} .
$$

Proof. If $G$ is the empty graph and if $G$ contains only one vertex the lemma is trivially true. The lemma can then be proved for $N$ larger than one by induction, assuming that the lemma holds for all complete graphs, $G^{\prime}$, with $N\left(G^{\prime}\right) \leqq N-1$. For $Q(G ; x)$ the recurrence relation (4.1) takes the form

$$
Q(G ; x)=x Q(G-i ; x)-\sum_{j \in G-i} W(i, j) Q(G-i-j ; x) .
$$

Here $Q(G-i ; x)$ is polynomial of degree $N-1$ and the sum-polynomial

$$
\sum_{j \in G-i} W(i, j) Q(G-i-j ; x),
$$

is polynomial of degree $N-2$. From the induction assumption it follows that the zeros of $Q(G-i-j ; x)$ interlace the zeros of $Q(G-i ; x)$ for all $j \in G-i$. Since $W(i, j)$ is positive for all $j \in G-i$ then the zeros of the sumpolynomial (4.4) will also interlace the zeros of $Q(G-i, x)$. Now, considering the sign of the right-hand side of (4.3) as $x$ takes the values of the zeros of $Q(G-i ; x)$ together with the sign as $x$ approaches $+\infty$ and $-\infty$ (see Fig. 1), one easily concludes that $Q(G ; x)$ has $N$ real zeros which

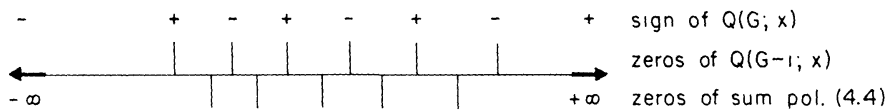

Fig. 1. 
satisfy (4.2). Since $Q(G ; x)$ is a polynomial of degree $N$ it does not have any other zeros.

By a continuity argument one can easily extend the lemma to include the case where some edge weights are zero and obtain the following theorem.

Theorem 4.2. For any weighted graph, $G, Q(G ; x)$ has only real zeros. Further, if $i$ is any vertex in $G$ and if $a_{1}, a_{2}, \ldots, a_{N}$ are the zeros of $Q(G ; x)$ while the zeros of $Q(G-i ; x)$ are $a_{1}^{\prime}, a_{2}^{\prime}, \ldots, a_{N-1}^{\prime}$, then

$$
a_{1} \leqq a_{1}^{\prime} \leqq a_{2} \leqq a_{2}^{\prime} \leqq \cdots \leqq a_{N-1}^{\prime} \leqq a_{N} .
$$

Remark 4.1. Theorem 4.2 does, of course, imply that the zeros of $P(G ; x)$ are purely imaginary.

Remark 4.2. One might be interested in knowing when the inequalities in (4.5) will all be strict, especially since strict interlacing implies distinct zeros. Example 4 in Section III shows that connectedness is not sufficient to ensure distinct zeros. One can also construct counterexamples to the conjecture that the absence of articulation points would be sufficient to ensure distinct zeros (see Fig. 2). Instead we propose the following amendment which, although it does not exhaust all the possibilities, covers most cases of interest.

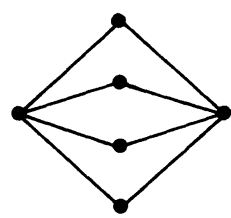

(a)

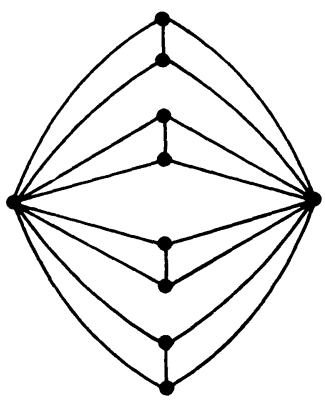

(b)

Fig. 2.

Amendment to Theorem 4.2. If $G$ has a Hamilton walk which ends at the i'th vertex then all the inequalities in (4.5) are strict.

Proof. As for Lemma 4.1, the proof inductively uses the recurrence relation (4.3). The important thing to notice is that if $G-i$ contains at least one vertex, $k$, such that $W(i, k)>0$ and such that the zeros of $Q(G-i ; x)$ and $Q(G-i-k ; x)$ obey the strict interlacing property, then 
the zeros of $Q(G-i ; x)$ and the sum-polynomial (4.4) will also obey the strict interlacing property. Consequently the strict interlacing property is retained along the Hamilton Walk.

Definition 4.1. For any graph, $G$, and any vertex, $j$, in $G$ we define the vertex sum $W_{j}$ by

$$
W_{j}=\sum_{i \in G-j} W(i, j)-\min \{W(i, j): i \in G, W(i, j)>0\} .
$$

We further define the numbers $B_{1}, B_{1}^{\prime}$ and $B_{1}^{\prime \prime}$ by

and

$$
\begin{aligned}
& B_{1}^{\prime}=\max \left\{W_{j}: j \in G\right\}, \\
& B_{1}^{\prime \prime}=\frac{1}{4} \max \{W(i, j) ; i \in G, j \in G\}
\end{aligned}
$$

$$
B_{1}=\max \left\{B_{1}^{\prime}, B_{1}^{\prime \prime}\right\} \text {. }
$$

Theorem 4.3. The (real) zeros of $Q(G ; x), a_{1}, a_{2}, \ldots, a_{N}$, satisfy

$$
\left|a_{i}\right|<2 \sqrt{B_{1}} \text {, all } i \text {. }
$$

Proof. If $G$ does not have a component which contains only two vertices then clearly $B_{1}^{\prime \prime}<B_{1}^{\prime}$. Since the bound on the zeros imposed by $B_{1}^{\prime \prime}$ is correct for a graph containing only two vertices we can concentrate on proving that $2 \sqrt{B_{1}^{\prime}}$ is a bound for the zeros when $G$ is a connected graph consisting of at least three vertices. For this purpose we shall need one more definition and a lemma:

Definition 4.2. Given a connected, weighted graph, $G$, we construct a (not necessarily unique) set, $\mathscr{E}_{G}$, of ordered pairs of section graphs of $G$ such that $\mathscr{E}_{G}$ contains the following members:

1) The pair $(G-1, G-1-i)$ is a member of $\mathscr{E}_{G}$ if $W(1, i) \neq 0$. Since $G$ is connected there is at least one pair of this type in $\mathscr{E}_{G}$.

2) If $\left(G^{\prime}, G^{\prime}-i\right)$ is a member of $\mathscr{E}_{G}$ then $\left(G^{\prime}-i, G^{\prime}-i-j\right)$ is a member whenever $W(i, j) \neq 0$ and $j \in G^{\prime}-i$.

3) If $\left(G^{\prime}, G^{\prime}-i\right)$ is a member of $\mathscr{E}_{G}$ such that $W(i, j)=0$ for all $j$ in $G^{\prime}-i$, then choose a vertex, $j$, in $G^{\prime}-i$ for which at least one of the neighbors in $G$ does not belong to $G^{\prime}-i$ and include $\left(G^{\prime}-i, G^{\prime}-i-j\right)$ as a member of $\mathscr{E}_{G}$.

Lemma 4.4. If $G$ is a connected graph, $\left(G^{\prime}, G^{\prime}-i\right) \in \mathscr{E}_{G}$ and $x \geqq 2 \sqrt{B_{1}^{\prime}}$ then $Q\left(G^{\prime} ; x\right) / Q\left(G^{\prime}-i ; x\right) \geqq \sqrt{B_{1}^{\prime}}$ and $Q\left(G^{\prime}, x\right)>0$, provided $G^{\prime}$ is not $G$.

Proof. Consider again the fundamental recurrence relation:

$$
Q\left(G^{\prime} ; x\right)=x Q\left(G^{\prime}-i ; x\right)-\sum_{j \in G^{\prime}-i} W(i, j) Q\left(G^{\prime}-i-j ; x\right) .
$$

If $\left(G^{\prime}, G^{\prime}-i\right) \in \mathscr{E}_{G}$ then it follows from the definition of $\mathscr{E}_{G}$ that

$$
\sum_{j \in G^{\prime}-i} W(i, j) \leqq B_{1}^{\prime}
$$


Table 1. Comparison between bound for the zeros (Theorem 4.3) and the actual value of the largest zero

\begin{tabular}{lll}
\hline Lattice & $\begin{array}{l}\text { Largest zero } \\
\text { squared }^{\mathrm{a}}\end{array}$ & $4 B_{1}$ \\
\hline Simple quadratic & 11.24 & 12 \\
Plane triangular & 17.86 & 20 \\
Tetrahedral & 11.76 & 12 \\
Simple cubic & 19.23 & 20 \\
Body-centered cubic & 26.81 & 28 \\
Face-centered cubic & 41.32 & 44 \\
\hline
\end{tabular}

a $-1 / r$ as given by Eq. (3.1) in Gaunt [28].

and that the sum over $j$ in (4.11) can be confined to $j$ 's for which $\left(G^{\prime}-i, G^{\prime}-i-j\right) \in \mathscr{E}_{G}$. Hence, if one divides Eq. (4.11) by $Q\left(G^{\prime}-i ; x\right)$ one can easily prove the lemma by induction.

Proof of Theorem 4.3. (cont.) The only step missing in the lemma above is the last step where $G^{\prime}$ in Eq. (4.11) is identical to G. Also in this case one can confine the sum over $j$ to values for which $\left(G^{\prime}-i, G^{\prime}-i-j\right) \in \mathscr{E}_{G}$. Using

$$
\sum_{j=2}^{N} W(1, j) \leqq 2 B_{1}^{\prime}
$$

one can then furnish this final step in the proof by showing that Lemma 4.4 with $G^{\prime} \equiv G-1$ implies that the right hand side of Eq. (4.3) is nonnegative and strictly positive if $x>2 \sqrt{B_{1}}$.

Remark 4.3. It is seen that the bound on the zeros given by Theorem 4.3 is the best possible for the four examples considered in Section III. This cannot be expected to be true in general, but that the bound is rather good, at least for graphs with equal edge weights, can be seen from Table 1 where the bound given by Theorem 4.3 is compared with the value for the largest zero found numerically by Gaunt [28]. It is also interesting to compare the bound given by Theorem 4.3 with the bound which can be obtained by applying the general theory of Ruelle [67] to the system of hard dimers. $C(\beta)$ in Ruelle's notation is, in this case, given by

$$
C(\beta)=2 B_{4} \equiv \max _{\substack{[i, j] \in G \\ W(i, j)>0}}\left\{\sum_{\substack{h \neq i, j \\ h \in G}}[W(i, h)+W(j, h)+W(i, j)]\right\},
$$

while $B$ in Ruelle's notation is zero. One obtains $2 e B_{4}$ as an upper bound on the square of the zeros as compared to $4 B_{1}^{\prime}$ with the present theory.

Theorem 4.5. Assume that $G$ is a graph with complex edge weights and write $|G|$ for the graph obtained from $G$ by replacing the edge weights 
by their moduli. If $A$ is any upper bound on the zeros of $Q(|G| ; x)$ then $P\left(G ; x_{1}, x_{2}, \ldots, x_{N}\right)$ is not zero if $\left|x_{j}\right|>A$ for $1 \leqq j \leqq N$.

Proof. From Eq. (4.11) it follows that

$$
\begin{aligned}
& \left|\frac{P\left(G^{\prime} ; x_{1}, x_{2}, \ldots\right)}{P\left(G^{\prime}-i ; x_{1}, x_{2}, \ldots\right)}\right| \\
& \geqq\left|x_{i}\right|-\sum_{j \in G^{\prime}-i}|W(i, j)|\left|\frac{P\left(G^{\prime}-i ; x_{1}, x_{2}, \ldots\right)}{P\left(G^{\prime}-i-j ; x_{1}, x_{2}, \ldots\right)}\right|^{-1} .
\end{aligned}
$$

From this inequality one easily deduces inductively that

$$
\left|\frac{P\left(G^{\prime} ; x_{1}, x_{2}, \ldots\right)}{P\left(G^{\prime}-i ; x_{1}, x_{2}, \ldots\right)}\right| \geqq \frac{Q\left(\left|G^{\prime}\right| ; A\right)}{Q\left(\left|G^{\prime}-i\right| ; A\right)},
$$

if $x_{1}, x_{2}, \ldots, x_{N}$ and $A$ fullfill the condition of the theorem and $G^{\prime}$ is a section graph of $G$. The inequality (4.15) implies the theorem.

Theorem 4.6. Let $G$ be a weighted graph with $N$ vertices and $G^{\prime}$ a subgraph. If $\operatorname{Re}\left(x_{i}\right)>0$ for all $i \in G^{\prime}$ and $\operatorname{Re}\left(x_{i}\right)=0$ for all $i \in G-G^{\prime}$ then the polynomial $P\left(G ; x_{1}, \ldots, x_{N}\right)$ can only be zero if $P\left(G ; x_{1}, \ldots, x_{N}\right)$ is identically zero when $P\left(G ; x_{1}, \ldots, x_{N}\right)$ is considered as a polynomial in $\left\{x_{i}\right\}, i \in G^{\prime}$, with the other vertex weights $\left\{x_{i}\right\}, i \in G-G^{\prime}$, kept fixed at the given values. The same statement is true if $\operatorname{Re}\left(x_{i}\right)<0$ for all $i \in G^{\prime}$. Here we return to the case of real, positive edge weights and $G^{\prime}$ does not have to be a proper subgraph of $G$ because the only relevant fact about $G^{\prime}$ is the specification of its vertices.

Proof. Define the corresponding $Q$-polynomial:

$$
Q\left(G ; x_{1}, \ldots, x_{N}\right)=i^{-N} P\left(G ; i x_{1}, \ldots, i x_{N}\right) .
$$

We shall then prove the theorem by proving the following two lemmas: The first, Lemma 4.7, implies that the theorem is true with $G^{\prime}=G$, in which case the theorem simply says that $P\left(G ; x_{1}, \ldots, x_{N}\right)$ is not zero when $\operatorname{Re}\left(x_{i}\right)>0$ for $i=1, \ldots, N$. The second, Lemma 4.8, then allows one to generalize the theorem as follows: Suppose that the vertices in $G^{\prime}$ are $1, \ldots, k-1$ and that we wish to set $x_{j}=i \alpha_{j}$ for $j=k, \ldots, N$. Then the polynomial (and hence holomorphic function)

$$
P\left(G ; x_{1}, \ldots, x_{k-1}, i \alpha_{k}, \ldots, i \alpha_{N}\right)
$$

is equal to

$$
\lim _{x_{k} \rightarrow i \alpha_{k}} \ldots \lim _{x_{N} \rightarrow i \alpha_{N}} P\left(G ; x_{1}, \ldots, x_{N}\right), \operatorname{Re}\left(x_{J}\right)>0 .
$$


Lemma 4.7. If $\operatorname{Im}\left(x_{j}\right)<0$ for $1 \leqq j \leqq N$ and if $\left(G^{\prime}, G^{\prime}-i\right) \in \mathscr{E}_{G}$ then

and

$$
\operatorname{Im}\left\{Q\left(G^{\prime}-i ; x_{1}, x_{2}, \ldots\right) / Q\left(G^{\prime} ; x_{1}, x_{2}, \ldots\right)\right\}>0,
$$

$$
Q\left(G^{\prime} ; x_{1}, x_{2}, \ldots\right) \neq 0 \text {. }
$$

Proof. If $G^{\prime}$ contains only one vertex then the lemma clearly holds since one then has:

$$
Q\left(G^{\prime}-i ; x_{1}, x_{2}, \ldots\right) / Q\left(G^{\prime} ; x_{1}, x_{2}, \ldots\right)=1 / x_{i} .
$$

If $G^{\prime}$ contains more than one vertex then we use the recurrence relation (4.11) to obtain

$$
\begin{array}{r}
\operatorname{Im}\left\{Q\left(G^{\prime} ; x_{1}, x_{2}, \ldots\right) / Q\left(G^{\prime}-i ; x_{1}, x_{2}, \ldots\right)\right\}=\operatorname{Im}\left\{x_{i}\right\}-\sum_{j \in G^{\prime}-i} W(i, j) \\
\cdot \operatorname{Im}\left\{Q\left(G^{\prime}-i-j ; x_{1}, x_{2}, \ldots\right) / Q\left(G^{\prime}-i ; x_{1}, x_{2}, \ldots\right)\right\},
\end{array}
$$

and once again we can complete the proof by induction.

Lemma 4.8. Let $D$ be a connected open set in $\mathbb{C}^{n}$ and let $\left\{f_{j}\right\}$ be a sequence of holomorphic functions on $D$ with the following properties:

(i) The $f_{j}$ are uniformly bounded on compact subsets of $D$;

(ii) $\left\{f_{j}\right\}$ converges to a function $f$ pointwise on $D$;

(iii) For each $j$ and each $z \in D, f_{j}(z) \neq 0$.

Then the convergence is uniform on compact subsets of $D, f$ is holomorphic, and either $f \equiv 0$ on $D$ or else $f(z) \neq 0$ for all $z \in D$.

Proof. The uniform convergence on compacta and the analyticity of $f$ is Montel's Theorem. When $n=1$, the remainder of the lemma is Hurwitz's Theorem. If $n>1$ and if $f\left(z_{0}\right)=0$ for some $z_{0} \in D$, then Hurwitz's Theorem states that $f \equiv 0$ on $P \cap D$, where $P$ is any one-dimensional hyperplane through $z_{0}$. Hence $f \equiv 0$ on some polydisc containing $z_{0}$ and, consequently, $f \equiv 0$ on $D$.

One might wonder how Theorem 4.6 would change if one allowed complex edge weights and whether there is a general theorem that combines Theorems 4.5 and 4.6. We first remark that if the edge weights are of the simple form

$$
W(k, j)=|W(k, j)| \exp \left[i\left(\theta_{k}+\theta_{j}\right)\right],
$$

then we can use the correspondence given in Eqs. (2.10), (2.11) in reverse to obtain

$$
P\left(G ; x_{1}, \ldots, x_{N}\right)=P\left(|G| ; x_{1} \exp \left(-i \theta_{1}\right), \ldots, x_{N} \exp \left(-i \theta_{N}\right)\right),
$$

where $|G|$ again means that we have replaced the edge weights by their moduli. Theorems 4.5 and 4.6 are directly applicable in this case which may suffice for many purposes. There exists, however, a general theorem which allows the edge weights to vary independently of each other and 
we shall conclude this section with that Theorem 4.9. Essentially it sums up all the preceding theorems. First we need a definition of the types of regions of the complex plane which we are going to consider.

Definition 4.3. The closed circular disk $D_{-}(A, \theta)$ in the complex plane is defined for $A$ real and non-negative and $0 \leqq \theta \leqq \pi / 2$ as the closed subset of the complex plane:

$$
D_{-}(A, \theta)=\{x: x \in \mathbb{C},|x+A \cot \theta| \leqq A / \sin \theta\} .
$$

Similarly $D_{+}(A, \theta)$ is defined by

$$
D_{+}(A, \theta)=\{x: x \in \mathbb{C},|x-A \cot \theta| \leqq A / \sin \theta\} .
$$

$D_{ \pm}(A, \theta)$ is defined as the intersection of $D_{-}(A, \theta)$ and $D_{+}(A, \theta)$, i.e.

$$
\begin{gathered}
D_{ \pm}(A, \theta)=\{x: x \in \mathbb{C},|x+A \cot \theta| \leqq A / \sin \theta, \\
|x-A \cot \theta| \leqq A / \sin \theta\} .
\end{gathered}
$$

The boundary of $D_{-}(A, \theta)$ and the boundary of $D_{+}(A, \theta)$ include the points $x= \pm i A$. We write $D_{-}^{C}(A, \theta)$ for the complement of $D_{-}(A, \theta)$ with respect to the complex plane, and similarly for $D_{+}^{C}(A, \theta)$. Clearly $0 \in D_{ \pm}(A, \theta)$.

Theorem 4.9. Let $G$ be a graph with complex edge weights such that

$$
\begin{gathered}
W(i, j)=-U(i, j)^{2}, \\
U(i, j) \in D_{ \pm}(V(i, j), \theta), \quad \forall i, j \in G,
\end{gathered}
$$

with $V(i, j)$ real and non-negative and with $0 \leqq \theta \leqq \pi / 2$. Let $\bar{G}$ be the graph with positive edge weights $V(i, j)^{2}$. Then $P\left(G ; x_{1}, \ldots, x_{N}\right)$ is not zero if $x_{i} \in D_{-}^{C}(A, \theta)$, all $i \in G$; or if $x_{i} \in D_{+}^{C}(A, \theta)$, all $i \in G$, where $A$ is the largest zero of $Q(\bar{G} ; x)$.

Proof. The proof is by induction from the recurrence relations for a subgraph $G^{\prime}$ in the form

$$
\begin{aligned}
& \frac{P\left(G^{\prime} ; x_{1}, \ldots, x_{N}\right)}{P\left(G^{\prime}-i ; x_{1}, \ldots, x_{N}\right)} \\
= & x_{i}-\sum_{j \in G^{\prime}-i} U(i, j)^{2}\left[\frac{P\left(G^{\prime}-i ; x_{1}, \ldots, x_{N}\right)}{P\left(G^{\prime}-i-j ; x_{1}, \ldots, x_{N}\right)}\right]^{-1} .
\end{aligned}
$$

Choose $\delta>0$ and assume that $x_{i} \in D_{-}^{C}(A+\delta, \theta)$ for all $i$. The induction assumption is that

$$
\frac{P\left(G^{\prime} ; x_{1}, \ldots, x_{N}\right)}{P\left(G^{\prime}-i ; x_{1}, \ldots, x_{N}\right)} \in D_{-}^{C}\left(\frac{Q\left(\bar{G}^{\prime} ; A+\delta\right)}{Q\left(\bar{G}^{\prime}-i ; A+\delta\right)}, \theta\right),
$$

where $\bar{G}^{\prime}$ is related to $\bar{G}$ as $G^{\prime}$ is to $G$. The induction is easily established from the following three lemmas and since $\delta$ was arbitrary, the theorem is proved. 
Lemma 4.10. Let $A>0$ and $0 \leqq \theta \leqq \pi / 2$. Then $x \in D_{-}^{C}(A, \theta)$ if and only if $x^{-1} \in D_{+}\left(A^{-1}, \theta\right)$. The same is true if - and + are interchanged.

Proof. Trivial.

Lemma 4.11. If $x \in D_{ \pm}(A, \theta)$ and $y \in D_{+}(B, \theta)$ with $A \geqq 0, B \geqq 0$ and $0 \leqq \theta \leqq \pi / 2$, then $x^{2} y \in D_{-}\left(A^{2} B, \theta\right)$.

Proof. By the maximum modulus principle it is sufficient to prove the lemma when $x$ and $y$ are on the boundaries of their respective domains. By symmetry it is also sufficient to consider $\operatorname{Re}(x) \geqq 0$. This means that we want to consider points in the complex plane of the form

$$
\begin{gathered}
x^{2} y=A^{2} B\left(-\cot \theta+e^{i \phi} / \sin \theta\right)^{2}\left(\cot \theta+e^{i \eta} / \sin \theta\right), \\
-\theta \leqq \phi \leqq \theta ; \quad 0 \leqq \eta \leqq 2 \pi .
\end{gathered}
$$

We want to prove that

$$
\left|x^{2} y+A^{2} B \cot \theta\right| \leqq A^{2} B / \sin \theta,
$$

when $x^{2} y$ is given by (4.30). The condition (4.31) is equivalent to

$$
\left|\left(-\cos \theta+e^{i \phi}\right)^{2}\left(\cos \theta+e^{i \eta}\right)+\sin ^{2} \theta \cos \theta\right|^{2}-\sin ^{4} \theta \leqq 0 .
$$

By tedious computation one can transform the left side of (4.32) to

$$
8 \cos \theta(\cos \theta-\cos \phi)\left(\cos \theta \sin \frac{\phi-\eta}{2}+\sin \frac{\phi+\eta}{2}\right)^{2} .
$$

Since $-\theta \leqq \phi \leqq \theta \leqq \pi / 2$ implies that $\cos \theta \leqq \cos \phi$, (4.32) is satisfied.

Lemma 4.12. If $x \in D_{-}^{C}(A, \theta)$ and $y \in D_{-}(B, \theta)$ with $A \geqq B \geqq 0$ and $0 \leqq \theta \leqq \pi / 2$, then $x-y \in D_{-}^{C}(A-B, \theta)$.

Proof. Follows trivially from the triangle inequality.

\section{An Alternative Proof of Theorem 4.9 Using Analytic Function Theory}

By the above, rather involved analysis of the recurrence relation we were able to prove Theorem 4.9 which can be thought of as interpolating between Theorems 4.5 and 4.6. It is a remarkable conclusion of the theory of analytic functions of several complex variables, however, that Theorems 4.5 and 4.6 automatically imply Theorem 4.9 without regard to the specific nature of the function $P\left(G ; x_{1}, \ldots, x_{N}\right)$. Since the powerful concept of a domain of holomorphy in $\mathbb{C}^{N}$ has heretofore rarely been used in statistical mechanics, the following alternative proof of Theorem 4.9 may have some value.

Instead of proving that $P$ has no zeros we shall prove that $f \equiv 1 / P$ is analytic. We suppose there are $M$ different edge weights which we label 
as $W_{1}, \ldots, W_{M}$ and define new variables $y_{1}, \ldots, y_{M}$ by

$$
\exp \left(i y_{j}\right)=\frac{i-U_{j} / V_{j}}{i+U_{j} / V_{j}}, \quad j=1, \ldots, M,
$$

where the U's and $V$ 's are defined in (4.26) and (4.27). Clearly $y_{j}$ is holomorphic in $U_{j} / V_{j}$ except on the cuts $(i, i \infty)$ and $(-i,-i \infty)$. The curve in the $U_{j}$ plane defined by $\operatorname{Re}\left(y_{j}\right)=$ constant is a circular arc with end points $+i V_{j}$ (corresponding to $\operatorname{Im}\left(y_{j}\right)=\infty$ ) and $-i V_{j}$ (corresponding to $\left.\operatorname{Im}\left(y_{j}\right)=-\infty\right)$. In fact, for $0 \leqq \theta \leqq \pi / 2$, the intersection of the boundary of the disc $D_{+}\left(V_{j}, \theta\right)$ with the right hand plane is the curve $\operatorname{Re}\left(y_{j}\right)=\pi-\theta$ while the intersection of the boundary of $D_{+}\left(V_{j}, \theta\right)$ with the left hand plane is the curve $\operatorname{Re}\left(y_{j}\right)=-\theta$ and similarly for $D_{-}\left(V_{j}, \theta\right)$. The domain $D_{ \pm}\left(V_{j}, \theta\right)$ maps onto the domain $\left|\operatorname{Re}\left(y_{j}\right)\right|<\theta$.

For the activity variables we first make the replacement $x_{j} \rightarrow 1 / t_{j}$, $j=1, \ldots, N$ and then consider the polynomial

$$
\tilde{P} \equiv\left[\prod_{j=1}^{N} t_{j}\right] P\left(G ; t_{1}^{-1}, \ldots, t_{N}^{-1}\right)
$$

which is analytic in a neighborhood of $t_{1}=t_{2}=\cdots=t_{N}=0$. Recalling that $A \geqq 0$ is the largest zero of $Q(\bar{G} ; x)$ we define new variables $z_{1}, \ldots, z_{N}$ by

$$
\exp \left(i z_{j}\right)=\frac{i-A t_{j}}{i+A t_{j}}, \quad j=1, \ldots, N .
$$

Let us consider the complex numbers $Y=\left(y_{1}, \ldots, y_{M}\right)$ together with $Z=\left(z_{1}, \ldots, z_{N}\right)$ as a point in $\mathbb{C}^{M+N}$ and define $f: \mathbb{C}^{M+N} \rightarrow \mathbb{C}$ by $f(Y, Z)$ $\equiv 1 / \tilde{P}$. Then Theorem 4.5 can be rephrased as:

$f$ is holomorphic on the tube, $T$, with real base

$$
\begin{aligned}
& \left|\operatorname{Re}\left(y_{j}\right)\right|<\pi / 2, \quad j=1, \ldots, M \quad \text { and } \\
& \left|\operatorname{Re}\left(z_{j}\right)\right|<\pi / 2, \quad j=1, \ldots, N .
\end{aligned}
$$

Likewise, Theorem 4.6 can be rephrased as:

$f$ is holomorphic on the tubes $T_{+}$and $T_{-}$with real bases

$$
\begin{gathered}
\operatorname{Re}\left(y_{j}\right)=0, \quad j=1, \ldots, M \text { and for } \\
j=1, \ldots, N, \quad 0 \leqq \operatorname{Re}\left(z_{j}\right)<\pi \text { for } T_{+} \text {while } \\
-\pi<\operatorname{Re}\left(z_{j}\right) \leqq 0 \quad \text { for } T_{-} .
\end{gathered}
$$

As $D=T \cup T_{+} \cup T_{-}$is connected, the Tube Theorem states that the envelope of holomorphy of $D$ is the domain $\operatorname{ch}(D)$ where $\operatorname{ch}()$ means convex hull. That is, every function that satisfies (4.35) and (4.36) has a holomorphic extension to $\operatorname{ch}(D)$. We claim that $\operatorname{ch}(D)$ is precisely the 
domain given by Theorem 4.9 , i.e.

where

$$
\operatorname{ch}(D)=\bigcup_{0 \leqq \theta \leqq \pi / 2}\left[K_{\theta}^{+} \cup K_{\theta}^{-}\right]
$$

$$
\begin{aligned}
K_{\theta}^{+}= & \left\{(Y, Z):\left|\operatorname{Re}\left(y_{j}\right)\right|<\theta, j=1, \ldots, M,-\theta<\operatorname{Re}\left(z_{j}\right)<\pi-\theta,\right. \\
& j=1, \ldots, N\} \\
K_{\theta}^{-}= & \left\{(Y, Z):\left|\operatorname{Re}\left(y_{j}\right)\right|<\theta, j=1, \ldots, M, \theta-\pi<\operatorname{Re}\left(z_{j}\right)<\theta, j=1, \ldots, N\right\} .
\end{aligned}
$$

To see that $K_{\theta}^{+}$, for example, is in $\operatorname{ch}(D)$ we let $\alpha$ denote the subset of $\{1, \ldots, N\}$ for which $\operatorname{Re}\left(z_{j}\right) \leqq 0$ and let $\beta$ be the complement of $\alpha$. Then $(Y, Z) \in K_{\theta}^{+}$means that $\operatorname{Re}\left(y_{j}\right)=\gamma_{j} \theta$, with $\left|\gamma_{j}\right|<1, j=1, \ldots, M, \operatorname{Re}\left(z_{j}\right)$ $=\delta_{j}(\pi-\theta), j \in \alpha$ and $\operatorname{Re}\left(z_{j}\right)=-\delta_{j} \theta, j \in \beta$ with $0 \leqq \delta_{j}<1$. We then form a convex combination of a point in $T$ with a point in $T_{+}$, i.e.

$(Y, Z)=\frac{2 \theta}{\pi}\left(Y_{1}, Z_{1}\right)+\left(1-\frac{2 \theta}{\pi}\right)\left(Y_{2}, Z_{2}\right)$,

with

$$
\begin{aligned}
& Y_{1}=\left(\gamma_{1} \frac{\pi}{2}, \ldots, \gamma_{M} \frac{\pi}{2}\right) \\
& Z_{1}=\left(S_{1} \delta_{1} \frac{\pi}{2}, \ldots, S_{N} \delta_{N} \frac{\pi}{2}\right), S_{j}=1, j \in \alpha ; S_{j}=-1, j \in \beta \\
& Y_{2}=(0, \ldots, 0) \\
& Z_{2}=\left(S_{1} \delta_{1} \pi, \ldots, S_{N} \delta_{N} \pi\right), S_{j}=1, j \in \alpha ; S_{j}=0, j \in \beta .
\end{aligned}
$$

Thus, we have recovered Theorem (4.9). The only thing that remains to be shown is that $\operatorname{ch}(D)$ is not larger than the domain given by the right side of (4.37). It is clear that $L_{+} \equiv \operatorname{ch}\left(T \cup T_{+}\right)=\bigcup_{0 \leqq \theta<\pi / 2} K_{\theta}^{+}$and similarly for $L_{-} \equiv \operatorname{ch}\left(T \cup T_{-}\right)$. We claim that $\operatorname{ch}(D)=L_{+} \cup L_{-}$. Since $T, T_{+}$and $T_{-}$ are all convex it follows easily that if there is a point in $\operatorname{ch}(D)$ not in $L_{+} \cup L_{-}$then there must be a point in $\operatorname{ch}(D)$ which is in $\operatorname{ch}\left(T_{+} \cup T_{-}\right)$and which is not in $L_{+} \cup L_{-}$. A point $(Y, Z)$ in $\operatorname{ch}\left(T_{+} \cup T_{-}\right)$has the property that $Y=(0, \ldots, 0)$ and $\left|z_{i}-z_{j}\right|<\pi$ for all $i, j=1, \ldots, N$. However, it is easy to see that $L_{+} \cup L_{-}$contains all such points, which completes the proof.

\section{Relationship of the Monomer-Dimer System to the Ising Model and to the Heisenberg Ferro- and Antiferromagnet}

Fisher [68] has shown how the Ising model with zero magnetic field can be put into a one to one correspondence with the dimer coverings of a suitably chosen weighted graph. As we shall show, that method can 
easily be extended to the Ising model with non-zero magnetic field, which can be put into a one to one correspondence with the monomer-dimer problem in which some of the vertices have zero monomer weight. One proceeds as follows: The Ising partition function corresponding to a weighted graph, $\hat{G}$, with edge weights $\left\{K_{i j}\right\}$, and $N$ vertices can be written as

$$
Z\left(\hat{G} ; z_{1}, z_{2}, \ldots, z_{N}\right)=\sum_{s= \pm 1}^{\hat{G}} \exp \left[\sum_{[i, j] \in \hat{G}} K_{i j}\left(s_{i} s_{j}-1\right)\right] \prod_{i=1}^{N} z_{i}^{s_{2}} .
$$

To each vertex, $i$, is associated a spin-variable, $s_{i}$, and a fugacity, $z_{i}$. The first sum runs over all values of the spin variables, and it is assumed that different vertices have different fugacities. With $s_{i}$ and $s_{j}$ equal to +1 or -1 one has the identities:

$$
\begin{aligned}
\exp \left(K_{i j} s_{i} s_{j}\right) & =\cosh \left(K_{i j}\right)\left[1+V_{i j} s_{i} s_{j}\right], \\
V_{i j} & =\tanh \left(K_{i j}\right), \\
z_{i}^{s_{i}} & =t_{i}\left(1+y_{i} s_{i}\right), \\
t_{i} & =\frac{1}{2}\left(z_{i}+z_{i}^{-1}\right), \\
y_{i} & =\left(z_{i}-z_{i}^{-1}\right) /\left(z_{i}+z_{i}^{-1}\right) .
\end{aligned}
$$

These can be used to obtain an alternative form of (5.1):

$$
\begin{aligned}
& Z\left(\hat{G} ; z_{1}, z_{2}, \ldots, z_{N}\right)= {\left[\prod_{[i, j] \in \hat{G}} \cosh \left(K_{i j}\right) \exp \left(-K_{i j}\right)\right] 2^{N} \prod_{i=1}^{N} t_{i} } \\
& \cdot \Upsilon\left(G ; y_{1}, y_{2}, \ldots, y_{N}\right), \\
& \Upsilon\left(G ; y_{1}, y_{2}, \ldots, y_{N}\right)=2^{-N} \sum_{s= \pm 1}^{G}\left[\prod_{[i, j] \in G}\left(1+V_{i j} s_{i} s_{j}\right)\right] \prod_{i=1}^{N}\left(1+y_{i} s_{i}\right) .
\end{aligned}
$$

Here, $G$ is the weighted graph with edge weights $\left\{V_{i j}\right\}$, vertex weights $\left\{y_{i}\right\}$ and $N$ vertices. It is important to notice that $G$ has no negative edge weights if and only if $\hat{G}$ has the same property. This latter condition means that $\hat{G}$ is an Ising ferromagnet. $\Upsilon\left(G ; y_{1}, y_{2}, \ldots, y_{N}\right)$ is essentially a partition function for walks on $G$ such that no edge is visited more than once.

The next step is to construct the expanded graph, $G^{E}$ : For each vertex, $i$, in $G$ with coordination number, $q(i)$, larger than one, $q(i)$ new vertices, $i_{1}, i_{2}, \ldots, i_{q(i)}$, are substituted. The edges which were incident on $i$ in $G$ become edges in $G^{E}$ with the same weight and incident on $\left\{i_{2}, i_{3}, \ldots, i_{q(i)}\right\}$ such that $i_{2}, i_{3}, \ldots, i_{q(i)-1}$ get one edge each while $i_{q(i)}$ gets two edges. Finally edges with weight one are added between $\left[i_{1}, i_{2}\right]$, 
$\left[i_{2}, i_{3}\right], \ldots,\left[i_{q(i)-1}, i_{q(1)}\right]$. The vertex weight of $i_{1}$ in $G^{E}$ is set equal to $y_{i}$ while $i_{2}, i_{3}, \ldots, i_{q(i)}$ are given vertex weight zero. Vertices in $G$ with coordination number one are transferred unaltered. Numbering the vertices in $G^{E}$ such that $i_{1}$ in the above notation gets number $i$ one finds

$$
\Upsilon\left(G ; y_{1}, \ldots, y_{N}\right)=\Upsilon\left(G^{E} ; y_{1}, \ldots, y_{N}, 0,0, \ldots, 0\right) .
$$

It is important that the vertices in $G^{E}$ have coordination number at most 3 and that vertices with non-zero value of the vertex weight have coordination number one.

The terminal graph, $\left(G^{E}\right)^{T}$, of $G^{E}$ can now be constructed: For each vertex, $i$, of $G^{E}$ with coordination number $q(i)$ a cluster of $q(i)$ new vertices is substituted; each of these is connected to the other $q(i)-1$ vertices by edges (called internal edges) with edge weight one. The vertex weight of the new vertices in $\left(G^{E}\right)^{T}$ are all taken to be equal to the vertex weight of the corresponding vertex in $G^{E}$. The edges which were incident on the vertex, $i$, in $G^{E}$ become edges incident on the vertices of the corresponding cluster in $\left(G^{E}\right)^{T}$, such that each of the $q(i)$ vertices gets connected by one edge. These edges are called external edges in $\left(G^{E}\right)^{T}$; their edge weights are taken to be the reciprocal of the edge weights of the corresponding edges in $G^{E}$.

Finally, it will be shown that $P\left(\left(G^{E}\right)^{T} ; y_{1}, \ldots, y_{N}, 0, \ldots, 0\right)$ and $\Upsilon\left(G^{E} ; y_{1}, \ldots, y_{N}, 0, \ldots, 0\right)$ can be expanded in such a manner that a one to one correspondence between non-zero terms in the expansions of the two partition functions obtains and that, furthermore, corresponding terms only differ by a factor which is the same for all terms. $P\left(\left(G^{E}\right)^{T} ; y_{1}, \ldots, y_{N}, 0, \ldots, 0\right)$ is expanded as a sum over all dimer arrangements (see Eq. (2.9)). For $r\left(G^{E} ; y_{1}, \ldots, y_{N}, 0, \ldots, 0\right)$ one takes the definition (5.8), expands the two products, and sums over all values of the spin variables. Corresponding to the term 1 in the factor $\left(1+V_{i j} s_{i} s_{j}\right)$ in Eq. (5.8), a dimer is placed on the corresponding external edge in $\left(G^{E}\right)^{T}$, while the term $V_{i j} s_{i} s_{j}$ corresponds to the external edge being free of a dimer. In the expansion of the second product in Eq. (5.8), the term $y_{i} s_{i}$ corresponds to a monomer on the analogous vertex in $\left(G^{E}\right)^{T}$ which otherwise must be covered by a dimer. Having kept the important properties of $G^{E}$ in mind, it is easily perceived that

$$
\begin{aligned}
r\left(G^{E} ; y_{1}, \ldots, y_{N}, 0, \ldots, 0\right) \\
\quad=\left[\prod_{\langle i, j\rangle \in G} V_{i, j}\right] P\left(\left(G^{E}\right)^{T} ; y_{1}, \ldots, y_{N}, 0, \ldots, 0\right) .
\end{aligned}
$$

This last polynomial, considered as a polynomial in $\left(y_{1}, \ldots, y_{N}\right)$ is not identically zero since otherwise the original Ising polynomial, (5.7), 
would be identically zero. Thus, the conditions of Theorem 4.6 are satisfied and we can conclude that $P\left(\left(G^{E}\right)^{T} ; y_{1}, \ldots, y_{N}, 0, \ldots, 0\right)$, as a polynomial in $\left(y_{1}, \ldots, y_{N}\right)$, satisfies Theorem 4.6. Since Eqs. (5.6) implies that $\left|z_{i}\right|<1$ is equivalent to $\operatorname{Re}\left(y_{i}\right)>0$ while $\left|z_{i}\right|>1$ is equivalent to $\operatorname{Re}\left(y_{1}\right)<0$. then Theorem 4.6 implies the Lee-Yang circle theorem for the Ising ferromagnet.

On the other hand, by taking the high-temperature limit of the Isingpartition function, one can prove that the Lee-Yang circle theorem implies the fact that the zeros of the monomer-dimer partition function are purely imaginary. For convenience we write the Ising partition function as

$$
Z(G ; z)=\left(z+z^{-1}\right)^{-N} \sum_{s= \pm 1}^{G} \exp \left(\beta \sum_{[i, j] \in G} J_{i j} s_{i} s_{j}\right) z^{\sum^{i=1} s_{i}},
$$

and, in contrast to what we did before, take the edge weights of the graph $G$ to be the $J_{i j}$ 's ( $J_{i j} \geqq 0$ corresponding to the ferromagnet). Introducing the variable $x$ defined by

$$
x=\beta^{-\frac{1}{2}}\left(z+z^{-1}\right)\left(z-z^{-1}\right)^{-1},
$$

one obtains the relation

$$
\left(z+z^{-1}\right)^{-1} z^{s_{2}}=\frac{1}{2 x}\left(x+s_{i} \beta^{-\frac{1}{2}}\right) .
$$

When this is substituted into Eq. (5.11) one gets

$$
Z(G ; z)=(2 x)^{-N} \sum_{s= \pm 1}^{G} \exp \left(\beta \sum_{[i, j] \in G} J_{i j} s_{i} s_{j}\right) \prod_{i=1}^{N}\left(x+s_{i} \beta^{-\frac{1}{2}}\right) .
$$

$Z(G ; z)$ is now expanded in powers of $\beta$. It is not difficult to see that the term of lowest order in $\beta$ is of zero'th order in $\beta$ and that it is precisely the monomer-dimer partition function for $G$. Consequently,

$$
(2 x)^{N} Z(G ; z)=P(G ; x)+0(\beta) .
$$

Since $|z|=1$ corresponds to purely imaginary $x$, the Lee-Yang circle theorem implies that the left-hand side of (5.15) considered as a polynomial in $x$ has purely imaginary zeros for all values of $\beta>0$. If one assumed that $P(G ; x)$ had a zero away from the imaginary axis one could easily prove from continuity of the zeros that for sufficiently small, but nonzero, $\beta$ the total right hand side of Eq. (5.15) would also have a zero outside the imaginary axis and one would thus have a contradiction. Consequently, the zeros of $P(G ; x)$ are all purely imaginary. Thus, by starting with the Lee-Yang theorem, one can derive part of Theorem 4.2 but not the amendment. 
The proof just given will also work the other way provided the zeros of $P(G ; x)$ are all simple. This means that Theorem 4.1 implies directly that if $G$ has a Hamilton walk and if $\beta$ is sufficiently small, then the zeros of the corresponding Ising ferromagnet partition function lie on the unit circle, are simple, and fulfill an interlacing condition similar to Eq. (4.5).

One can easily convince oneself that (5.15) also holds when $Z(G ; z)$ is a Heisenberg ferromagnet partition function of the form:

$$
\begin{gathered}
Z(G ; z)=\left(z+z^{-1}\right)^{N} \operatorname{Tr}\left\{\exp (\beta H) z_{z^{i=1}}^{N} s_{t z}\right\}, \\
H=\sum_{[i, j] \in G}\left(J_{i j} s_{i z} s_{j z}\right) \\
+\left(\text { quadratic expression in } s_{1 x}, s_{2 x}, \ldots, s_{1 y}, s_{2 y}, \ldots\right) .
\end{gathered}
$$

Furthermore, if $J_{i j}$ is changed to $-J_{i j}$ in the formulas above one obtains an antiferromagnet in place of the ferromagnet and at the same time $P(G ; x)$ is changed to $Q(G ; x)$. Since $z^{2}$ negative and real corresponds to $x$ real, these considerations imply that for an antiferromagnet the zeros in $z^{2}$ are all on the negative real axis for sufficiently small $\beta$ when $G$ has a Hamilton walk. One should note that the considerations above do not yield a bound, independent of the size of $G$, on the range of $\beta$ in which the zeros have the stated properties.

Our result, that the zeros of the Heisenberg ferromagnet lie on the unit circle when $\beta$ is small, is overshadowed by the result of Asano [70] that the zeros lie on the unit circle for all $\beta>0$. Asano's result has been extended by Suzuki and Fisher [71].

\section{Christoffell-Darboux Type Formulas for Monomer-Dimer and Ising Systems}

The recursion formula (4.1) suggests that it might be worthwhile to make an investigation of the general theory of orthogonal polynomials in order to obtain ideas which can be applied with success to the theory of monomer-dimer systems. Unfortunately, most results for orthogonal polynomials are not derived from the recursion formulae; the only important exception seems to be the Christoffel-Darboux Formula. This will be the main theme of the present section.

Definition 6.1. A self-avoiding walk on a weighted graph, $G$, is an ordered set of vertices in $G$, such that no vertex appears more than once. The weight, $W(S)$, associated with the walk

$$
S=\left\{i_{1}, i_{2}, \ldots, i_{m+1}\right\},
$$


of length $m$, for $m>0$, is given by

$$
W(S)=\prod_{j=1}^{m} W\left(i_{j}, i_{j+1}\right) .
$$

For $m=0$ we take $W(S)=1$. We shall write $[S]$ for the self-avoiding walk, $S$, considered as a subgraph of $G$, and the set of all self-avoiding walks on $G$ which start at the vertex $i$ and have non-zero weight will be denoted $\mathscr{S}_{i}$.

Definition 6.2. We define the kernel, $K(G, i ; y, x)$ by

$$
K(G, i ; y, x)=\sum_{S \in \mathscr{Y}_{1}} W(S) Q(G-[S] ; x) Q(G-[S] ; \bar{y}),
$$

where $\bar{y}$ is the complex conjugate of $y$.

Theorem 6.1. For every vertex, $i$, in a weighted graph, $G$,

$$
(x-\bar{y}) K(G, i ; y, x)=[Q(G ; x) Q(G-i ; \bar{y})-Q(G ; \bar{y}) Q(G-i ; x)] \text {. }
$$

Proof. By induction from the recursion formula (4.3).

Theorem 6.1 is the Christoffel-Darboux formula for the monomerdimer partition function.

Corollary 6.2. For every vertex, $i$, in a weighted graph, $G$,

$$
K(G, i ; \bar{x}, x)=Q^{\prime}(G ; x) Q(G-i ; x)-Q(G ; x) Q^{\prime}(G-i ; x),
$$

where the prime denotes differentiation with respect to $x$.

Proof. Trivial.

Remark 6.1. Theorem 4.2 with the amendment can be obtained easily from Theorem 6.1 and Corollary 6.2: If $Q(G ; x)$ had a complex zero, $a$, then $\bar{a}$ would also be a zero of $Q(G ; x)$. Taking $y=x=a$ in Eq. (6.4) one arrives at a contradiction because every term in (6.3) is clearly nonnegative and real. If $G$ has a Hamilton walk, there is at least one nonzero term, namely when $[S]=G$. If $G$ does not have a Hamilton walk we can appeal to a continuity argument. The interlacing can then be proved from Eq. (6.5) by considering the sign of $Q(G-i ; x)$ for $x$ equal to the zeros of $Q(G ; x)$.

Theorem 6.3. Let $H$ be a proper, non-empty subgraph of $G$, let $j$ be a vertex in $G-H$, and let $\mathscr{S}_{j}(H)$ be the set of all self-avoiding walks which start at the vertex $j$ and end at some vertex in $H$ without visiting $H$ before. Then

$$
\begin{aligned}
Q(G ; x) & Q(G-H-j ; x)-Q(G-j ; x) Q(G-H ; x) \\
& =-\sum_{S \in \mathscr{S}_{J}(H)} W(S) Q(G-[S] ; x) Q(G-[S]-H ; x),
\end{aligned}
$$

where in $G-[S]-H$ it is understood that the vertex common to $[S]$ and $H$ is deleted only once. 
Proof. The proof again follows by a simple application of the recursion formula.

It is interesting that while the recursion formula for the monomerdimer system has no interesting analog for the Ising model, the ChristoffelDarboux formula, (6.4), does. If $G$ is a weighted graph with positive edge weights, $\left\{V_{i j}\right\}$, and if $H$ is a subset of vertices in $G$, then we define

$$
\begin{aligned}
\Upsilon(G, H ; z)= & 2^{-N(G)} \sum_{s= \pm 1}^{G}\left\{\left[\prod_{j \in H}\left(-i s_{j}\right) \mid\right.\right. \\
& \left.\cdot\left[\prod_{[j, k] \in G}\left(1+V_{j k} S_{j} s_{k}\right)\right]\left[\prod_{j=1}^{N} z^{s_{j}}\right]\right\},
\end{aligned}
$$

where $i$ is the imaginary unit. We shall allow the same vertex of $G$ to occur repeatedly in $H$, in which case the corresponding factor in $\prod_{j \in H}\left(-i s_{j}\right)$ should also be repeated. $\Upsilon(G, \emptyset ; z)$ is seen to be a version of the Ising partition function (see Eqs. (5.3)-(5.8)). In the following, $H+j$ denotes the union of the set $H$ and the vertex $j$.

Theorem 6.4. Let $G, H$ and $\Upsilon$ be as defined above and introduce

$$
\begin{aligned}
\Psi(G, H, j ; x, y)= & \Upsilon(G, H+j ; x) \Upsilon(G, H ; y) \\
& -\Upsilon(G, H+j ; y) \Upsilon(G, H ; x) .
\end{aligned}
$$

Then there exists an expansion of $\Psi$ :

$$
\begin{aligned}
\Psi(G, H, j ; x, y)= & \frac{1}{2 i}\left(\frac{x}{y}-\frac{y}{x}\right) \sum_{G^{\prime} \subseteq G} \sum_{H^{\prime} \subseteq G^{\prime}} W\left(G, H, j ; G^{\prime}, H^{\prime}\right) \\
& \cdot \Upsilon\left(G^{\prime}, H^{\prime} ; x\right) \Upsilon\left(G^{\prime}, H^{\prime} ; y\right),
\end{aligned}
$$

such that the numbers $W\left(G, H, j ; G^{\prime}, H^{\prime}\right)$, are all non-negative when the edge weights $\left\{V_{j k}\right\}$ are all non-negative.

Proof. Expanding the factor $\left(1+V_{j k} s_{j} s_{k}\right)$ in Eq. (6.7) we obtain

$$
\begin{gathered}
\Upsilon(G, H+j ; z)=\Upsilon\left(G-V_{j k}, H+j ; z\right) \\
+V_{j k} \Upsilon\left(G-V_{j k}, H+k ; z\right), \\
\Upsilon(G, H ; z)=\Upsilon\left(G-V_{j k}, H ; z\right)-V_{j k} \Upsilon\left(G-V_{j k}, H+j+k ; z\right),
\end{gathered}
$$

where we have used $G-V_{j k}$ to denote the graph $G$ with the edge $[j, k]$ deleted. Then,

$$
\begin{aligned}
\Psi(G, H, j ; x, y)= & \Psi\left(G-V_{j k}, H, j ; x, y\right) \\
& +V_{j k} \Psi\left(G-V_{j k}, H, k ; x, y\right) \\
& +V_{j k} \Psi\left(G-V_{j k}, H+j, k ; x, y\right) \\
& +V_{j k}^{2} \Psi\left(G-V_{j k}, H+k, j ; x, y\right) .
\end{aligned}
$$


If $j$ is a vertex in a weighted graph, $G^{\prime}$, which is not connected to any other vertex in $G^{\prime}$ then, upon summing over $s_{j}= \pm 1$, one obtains:

$$
\Psi\left(G^{\prime}, H, j ; x, y\right)=\frac{1}{2 i}\left(\frac{x}{y}-\frac{y}{x}\right) \Upsilon(G-j, H ; x) \Upsilon(G-j, H ; y) .
$$

This result holds even if $H$ contains $j$, but in that case one should delete $j$ from $H$ on the right side of (6.12). The theorem then follows by combining Eqs. (6.11) and (6.12).

Eq. (6.9) is the Christoffel-Darboux formula for the Ising model.

Theorem 6.5. The zeros of $\Upsilon(G, H ; z)$ lie on the unit circle (i.e. satisfy $|z|=1$ ) for all $G$ and $H$ when all the $V_{i j}$ are non-negative (ferromagnetic case). If $e^{i \phi_{1}}, e^{i \phi_{2}}, \ldots, e^{i \phi_{N}}$ are the ordered zeros of $\Upsilon(G, H ; z)$,

$$
-\pi<\phi_{1} \leqq \phi_{2} \leqq \cdots \leqq \phi_{N} \leqq \pi,
$$

and if $e^{i \psi_{1}}, e^{i \psi_{2}}, \ldots, e^{i \psi_{N}}$ are the zeros of $\Upsilon(G, H+j ; z)$ ordered in the same manner, then either

or

$$
\begin{aligned}
& \phi_{1} \leqq \psi_{1} \leqq \phi_{2} \leqq \psi_{2} \leqq \cdots \leqq \phi_{N} \leqq \psi_{N} \\
& \psi_{1} \leqq \phi_{1} \leqq \psi_{2} \leqq \phi_{2} \leqq \cdots \leqq \psi_{N} \leqq \phi_{N}
\end{aligned}
$$

If $G$ is connected then all the inequalities in (6.13) are strict.

Proof. From the definition (6.7)

$$
\begin{aligned}
\Upsilon\left(G, H ; z^{-1}\right) & =(-1)^{N(H)} \Upsilon(G, H ; z), \\
\Upsilon(G, H ; \bar{z}) & =(-1)^{N(H)} \overline{\Upsilon(G, H ; z) .}
\end{aligned}
$$

Let $x$ be a root of $Y(G, H ; x)=0$ and set $y=(\bar{x})^{-1}$ in (6.8). On the right side of (6.9) there will be at least one non-zero $W$, corresponding to $G^{\prime}=G-j$ and $H^{\prime}=H$, as can be seen from (6.11). Then, by using (6.14) and induction, the right side of (6.9) is non-zero unless $|x|=1$. The proof of the interlacing parallels that in Remark 6.1.

\section{Some Inequalities on the Compressibility and Other Quantities}

In this section we display some inequalities that can be derived from the fact that the roots of $Q(G ; x)$ are real and come in equal and opposite pairs (except for $x=0$ ). Theorem 7.1 is of theoretical interest and will be used in Section VIII. Among other inequalities we derive a lower bound on the compressibility and show in Theorem 7.6 that this is stronger than the bound that can be derived using Ginibre's general method for repulsive potentials [72]. 
Definition 7.1. Let $a_{1}, a_{2}, \ldots, a_{N}$ be the (real) zeros of $Q(G ; x)$ ordered such that

$$
a_{j}=-a_{N+1-j},
$$

(which is possible since $Q(G ; x)$ contains either only even powers or only odd powers of $x)$. We then define $b_{i} \geqq 0(i=1,2, \ldots, N)$ by

$$
b_{i}=a_{i}^{2} .
$$

The numbers $-b_{1}, \ldots,-b_{M}$ (with $\left.M=[N / 2]\right)$ are the zeros of $R(G ; x)$.

Theorem 7.1. The canonical partition function $Z_{d}$ satisfies the inequality:

$$
2 \ln Z_{d} \geqq \ln Z_{d-1}+\ln Z_{d+1}+\ln \frac{(M-d+1)(d+1)}{(M-d) d} .
$$

Remark 7.1. This inequality states that even for finite systems the free energy per unit volume is a strictly convex function of the dimer density.

Proof. If Newton's inequality is applied to $R(G ; x)$ which is a polynomial of degree $M$ having $M$ real zeros (according to Theorem 4.2), then one gets

$$
d(M-d) Z_{d}^{2}-(d+1)(M-d+1) Z_{d-1} Z_{d+1} \geqq 0,
$$

which trivially yields (7.1).

Definition 7.2. For a weighted graph, $G$, and activity, $x$, the monomer density, $\varrho_{m}$, is given by

$$
\varrho_{m}=x N(G)^{-1} d \ln P(G ; x) / d x=x N(G)^{-1} P^{\prime}(G ; x) / P(G ; x) .
$$

Theorem 7.2. If $G$ has at least one edge then the zeros of $d \varrho_{m} / d x$ satisfy

$$
\left|x^{2}+b_{1} / 2\right| \leqq b_{1} / 2
$$

where $-b_{1}$ is the smallest (largest modulus) zero of $R(G ; x)$. If $G$ has no edges then $d \varrho_{m} / d x$ vanishes identically. In particular $\operatorname{Re}\left(x^{2}\right) \leqq 0$ and $\left|x^{2}\right| \leqq b_{1}$.

Proof. With $y=x^{2}$ one has

$$
\varrho_{m}=1-N^{-1} \sum_{j=1}^{N} b_{j}\left(y+b_{j}\right)^{-1},
$$

since $-b_{j}, j=1,2, \ldots, M$, are the zeros of $R(G ; x)$. Differentiation gives

$$
x \frac{d \varrho_{m}}{d x}=\frac{2 y}{N} \sum_{j=1}^{N} \frac{b_{j}}{\left(y+b_{j}\right)^{2}} .
$$


Setting $y=r e^{i \theta} \neq 0$ one finds

$$
\operatorname{Im}\left\{e^{2 i \theta} \frac{N}{4 x} \frac{d \varrho_{m}}{d x}\right\}=\sin \theta \sum_{j=1}^{N} \frac{b_{j}^{2}\left(r+b_{j} \cos \theta\right)}{\left|y+b_{j}\right|^{4}} .
$$

If $x$ fails to satisfy condition (7.6) then $r+b_{j} \cos \theta$ is positive for all $j$. The theorem then follows because $b_{j}$ is real for all $j$ and nonzero for at least one value of $j$. (If $G$ does not contain any edges then $b_{j}=0$ for all $j$ and $d \varrho_{m} / d x=0$, identically.)

Remark 7.2. Clearly $\varrho_{m}$ is an increasing function of $x \in(0, \infty)$ and Theorem 7.1, together with the implicit function theorem, guarantees that $x$ is real analytic in $\varrho_{m}$. In the thermodynamic limit (whose existence we shall prove in sections VIII and IX) the zeros of $d \varrho_{m} / d x$ also satisfy Theorem 7.2 (by Vitali's theorem). This means that the thermodynamic limit of $N(G)^{-1} \ln P(G ; x)$ can be thought of as a real analytic function of $\varrho_{m}$. The following Theorems 7.3 and 7.4 complement this assertion by giving an explicit lower bound to $d \varrho_{m} / d x$, but by themselves they do not obviously guarantee analyticity of $x$ in $\varrho_{m}$ since they are concerned solely with real $x$.

Theorem 7.3. The following bounds on $\varrho_{m}$ and $x d \varrho_{m} / d x$ follow from Theorem 4.1:

$$
\begin{aligned}
\varrho_{m} & \geqq\left(1+B_{2} x^{-2}\right)^{-1}, \\
x d \varrho_{m} / d x & \geqq 2 x^{2}\left(1-\varrho_{m}\right)^{2} / B_{2}, \\
x d \varrho_{m} / d x & \leqq 2 \varrho_{m}\left(1-\varrho_{m}\right),
\end{aligned}
$$

for real, positive $x . B_{2}$ is given by

$$
B_{2}=2 N^{-1} \sum_{\langle i, j\rangle \in G} W(i, j) .
$$

Proof. Since $\frac{1}{2} N B_{2}$ is the coefficient of $x^{M-1}$ in $R(G ; x)$ one has that

$$
\left(1+B_{2} x^{-2}\right)=x^{-2} N^{-1} \sum_{j=1}^{N}\left(b_{j}+x^{2}\right) .
$$

Eq. (7.7) can alternatively be written

and

$$
\varrho_{m}=x^{2} N^{-1} \sum_{j=1}^{N}\left(b_{j}+x^{2}\right)^{-1},
$$

$$
1-\varrho_{m}=N^{-1} \sum_{j=1}^{N} \frac{b_{j}}{x^{2}+b_{j}} .
$$

The inequality (7.10) follows from (7.14) and (7.15) since

$$
\left[N^{-1} \sum_{j=1}^{N}\left(b_{j}+x^{2}\right)\right]\left[N^{-1} \sum_{j=1}^{N}\left(b_{j}+x^{2}\right)^{-1}\right] \geqq 1 .
$$


The two inequalities:

and

$$
\left(1-\varrho_{m}\right)^{2} \leqq B_{2} N^{-1} \sum_{j=1}^{N} \frac{b_{j}}{\left(x^{2}+b_{j}\right)^{2}},
$$

$$
\left(1-\varrho_{m}\right)^{2} \leqq N^{-1} \sum_{j=1}^{N} b_{j}^{2}\left(x^{2}+b_{j}\right)^{-2},
$$

can be obtained from Eq. (7.16) by applying Cauchy's inequality. The inequality (7.11) then follows by substituting (7.8) into (7.18), while (7.12) can be obtained from (7.19) since

$$
x \frac{d \varrho_{m}}{d x}=\frac{2}{N} \sum_{j=1}^{N} \frac{b_{j}}{\left(x^{2}+b_{j}\right)}-\frac{2}{N} \sum_{j=1}^{N} \frac{b_{j}^{2}}{\left(x^{2}+b_{j}\right)^{2}} .
$$

A lower bound on $x d \varrho_{m} / d x$ can be obtained in an entirely different way by viewing the system as a hard core dimer gas. One can then prove

Theorem 7.4. $x \frac{d \varrho_{m}}{d x} \geqq 2\left(1-\varrho_{m}\right)\left(1+2 B_{3} x^{-2}\right)^{-1}$, where

$$
B_{3}=\max \left\{\sum_{j \in G-\imath} W(i, j): i \in G\right\} .
$$

Following Ginibre [72] one first proves

Lemma 7.5. $d Z_{d}^{2} \leqq Z_{d-1}\left[(d+1) Z_{d+1}+2 B_{3} Z_{d}\right]$.

Proof of Lemma 7.5. One has:

$$
d Z_{d}=\sum_{\substack{D \in \mathscr{A} \\ \# D=d-1}} W(D) \sum_{\langle i, j\rangle \in G-[D]} W(i, j) .
$$

An application of Cauchy's inequality yields

$$
\begin{aligned}
\left(d Z_{d}\right)^{2} \leqq & \left.\sum_{\substack{D \in \mathscr{D} \\
\# D=d-1}} W(D)\right]\left[\sum_{\substack{D \in \mathscr{D} \\
\# D=d-1}} W(D)\left\{\sum_{\{i, j\rangle \in G-[D]} W(i, j)\right\}^{2}\right] \\
\leqq & Z_{d-1}\left[d(d+1) Z_{d+1}+\sum_{\substack{D \in \mathscr{D} \\
\# D=d-1}} W(D)\right. \\
& \left.\cdot \sum_{\langle i, j\rangle \in G-[D]} W(i, j)\left\{\sum_{k \in G}(W(i, k)+W(j, k))\right\}\right] \\
\leqq & Z_{d-1}\left[d(d+1) Z_{d+1}+d Z_{d} 2 B_{3}\right],
\end{aligned}
$$

which proves the lemma.

Proof of Theorem 7.4. If one introduces the notation

$$
\langle f(d)\rangle=\sum_{d=0}^{M} f(d) Z_{d} x^{N-2 d} / P(G ; x),
$$


then one can write $\varrho_{m}=1-2 N^{-1}\langle d\rangle$ and

$$
x \frac{d \varrho_{m}}{d x}=4 N^{-1}\left\langle(d-\langle d\rangle)^{2}\right\rangle .
$$

If, furthermore, the inequality (7.22) is written as

$$
\begin{aligned}
& {\left[(d+1) ! Z_{d+1} x^{N-2(d+1)}\right]\left[d ! Z_{d} x^{N-2 d}\right]^{-1}} \\
& \quad \geqq\left[d ! Z_{d} x^{N-2 d}\right]\left[(d-1) ! Z_{d-1} x^{N-2(d-1)}\right]^{-1}-2 B_{3} x^{-2},
\end{aligned}
$$

then Ginibre's main theorem [72] implies

$$
\left\langle(d-\langle d\rangle)^{2}\right\rangle /\langle d\rangle \geqq\left(1+2 B_{3} x^{-2}\right)^{-1},
$$

which is equivalent to (7.21).

Theorem 7.4 is weaker than the lower bound on $x \frac{d \varrho_{m}}{d x}$ given in Theorem 7.3, Eq. (7.11). This can be seen from the following theorem:

Theorem 7.6. $\quad\left(1-\varrho_{m}\right) \geqq B_{2}\left(x^{2}+2 B_{3}\right)^{-1}$.

Proof. The inequality (7.29) may alternatively be written as

$$
2 B_{3} \geqq B_{2}\left(1-\varrho_{m}\right)^{-1}-x^{2} .
$$

From the inequality (7.11) one obtains

$$
\frac{d}{d x}\left(B_{2}\left(1-\varrho_{m}\right)^{-1}\right) \geqq 2 x,
$$

which implies that the right hand side of (7.30) is an increasing function of $x$. It will consequently be sufficient to prove (7.30) in the limit $x \rightarrow \infty$, i.e. to prove that

$$
2 B_{3} \geqq\left(N^{-1} \sum_{j=1}^{N} b_{j}^{2}\right)\left(N^{-1} \sum_{j=1}^{N} b_{j}\right)^{-1} .
$$

Lemma 7.5 gives for $d=1$

Since

$$
Z_{1}^{2}<2 Z_{2}+2 B_{3} Z_{1}
$$

$$
\begin{gathered}
Z_{1}=-\sum_{j=1}^{M}\left(-b_{j}\right)=\frac{1}{2} \sum_{j=1}^{N} b_{j}, \\
Z_{2}=\sum_{j=1}^{M} \sum_{i=j+1}^{M}\left(-b_{j}\right)\left(-b_{i}\right)=\frac{1}{2} Z_{1}^{2}-\frac{1}{4} \sum_{j=1}^{N} b_{j}^{2},
\end{gathered}
$$

then (7.32) follows from (7.33). 
Remark 7.3. In this section and in Section IV we have introduced four different numbers $B_{1}, B_{2}, B_{3}$ and $B_{4}$ which all involve the sum of the weights of the edges incident on one vertex. $B_{3}$ is the maximum value of the sum; $B_{2}$ is the average value of the sum; $B_{1}$ is the maximum when one edge is deleted from the sum, and $B_{4}$ is somewhere between $B_{3}$ and $B_{1}$. If all vertices are equivalent except for boundary points then one obtains $B_{2}=B_{3}$ in the limit of an infinite graph if the fraction of boundary vertices tends to zero. If, moreover, all edge weights are equal to $W$ and the coordination number is $q$ then in the limit of an infinite graph one has

$$
B_{2}=B_{3}=q W ; \quad B_{1}=(q-1) W ; \quad B_{4}=\left(q-\frac{1}{2}\right) W .
$$

\section{The Thermodynamic Limit for Monomer-Dimer Systems: Basic Properties}

Since the monomer-dimer problem can be considered as the problem of a hard core dimer gas, it is fairly obvious from the general results on thermodynamic limits that the limit exists when the weighted graph $G$ tends to infinity in a reasonable manner. We shall, nevertheless, give an explicit proof of the existence of the thermodynamic limit for the monomer-dimer problem, partly to obtain stronger results and partly to demonstrate a different method of proof.

Definition 8.1. A Weighted Lattice, (or simply Lattice), $L$, is an infinite, weighted graph imbedded in $\mathbb{R}^{v}$ such that if $\boldsymbol{a}=\left\{a_{1}, a_{2}, \ldots, a_{v}\right\}$ is any vector with integer components then $L$ is mapped onto itself if it is translated by $\boldsymbol{a}$. We also assume that every bounded subset of $\mathbb{R}^{v}$ contains only a finite number of vertices of $L$.

Definition 8.2. The lattice, $L$, will be said to have compact interaction if there exists a finite upper bound on the Euclidean length of the edges incident at any vertex.

Remark 8.1. In the following we shall assume that the lattice, $L$, has compact interaction and that the units of the Euclidean space are chosen such that if the edge $[i, j]$ is represented by a vector from the $i$ th vertex to the $j$ 'th vertex, and if $\boldsymbol{j}-\boldsymbol{i}=\left\{j_{1}-i_{1}, j_{2}-i_{2}, \ldots, j_{v}-i_{v}\right\}$,

$$
\max _{W(i, j)>0}\left\{\max _{1 \leqq k \leqq v}\left|j_{k}-i_{k}\right|\right\}<1 .
$$

This will ensure that the interactions (nonzero edges) do not extend beyond the neighboring unit cells.

Remark 8.2. In the following we shall only consider subgraphs of the lattice $L$. If not otherwise specified all subgraphs will be assumed to be section graphs. 
Remark 8.3. Unless otherwise specified, vectors will be assumed to have integer components.

Definition 8.3. The section graph $\Lambda(\boldsymbol{a})$ includes every vertex with coordinates $x_{1}, x_{2}, \ldots, x_{v}$ which satisfies

$$
0 \leqq x_{1}<a_{1}, \quad 0 \leqq x_{2}<a_{2}, \ldots, 0 \leqq x_{v}<a_{v} .
$$

The section graph $\Lambda_{\boldsymbol{n}}(\boldsymbol{a})$ is the graph $\Lambda(\boldsymbol{a})$ translated by $\left\{n_{1} a_{1}, n_{2} a_{2}, \ldots, n_{v} a_{v}\right\}$.

Definition 8.4. Let $\Lambda$ be any section graph of $L$. The number $N_{a}^{+}(\Lambda)$ is then defined as the number of graphs $\Lambda_{\boldsymbol{n}}(\boldsymbol{a})$ which, for a fixed and $\boldsymbol{n}$ running over all integer vectors, has at least one vertex in common with $\Lambda$. The number $N_{\boldsymbol{a}}^{-}(\Lambda)$ is defined similarly as the number of graphs $\Lambda_{\boldsymbol{n}}(\boldsymbol{a})$ which are section graphs of $\Lambda$.

Definition 8.5. Let $\{\Lambda\}$ be a sequence of finite graphs such that $N(\Lambda) \rightarrow \infty$. If

$$
\begin{aligned}
& \lim N_{a}^{-}(\Lambda) \rightarrow \infty, \\
& \lim N_{a}^{-}(\Lambda) / N_{a}^{+}(\Lambda) \rightarrow 1,
\end{aligned}
$$

for all $\boldsymbol{a}$ then the sequence is said to tend to infinity in the sense of Van Hove.

Remark 8.4. Whenever a sequence of section graphs of $L$ is said to tend to infinity it should always be understood to be in the sense of Van Hove.

Definition 8.6. $A_{0}(\Lambda)$ is the modulus of the zero of maximum modulus of $P(\Lambda ; x)$.

Definition 8.7. $\Lambda_{n} \equiv \Lambda\left(\left\{2^{n}-1,2^{n}-1, \ldots, 2^{n}-1\right\}\right) ; \Lambda_{n}+\boldsymbol{a}$ means the cube $\Lambda_{n}$ translated by the vector $\boldsymbol{a}$.

Lemma 8.1. The following limit exists:

$$
\lim _{n \rightarrow \infty} A_{0}\left(\Lambda_{n}\right)=A_{0}
$$

Proof. From Theorem 4.2, $A_{0}\left(\Lambda_{n}\right) \leqq A_{0}\left(\Lambda_{n+1}\right)$. From Theorem 4.3, $A_{0}\left(\Lambda_{n}\right)<2 \sqrt{B_{1}}$.

Theorem 8.2. If $\{\Lambda\}$ is a sequence of section graphs which tends to infinity then

$$
\lim _{\Lambda \rightarrow \infty} A_{0}(\Lambda)=A_{0} .
$$

Proof. Since $A$ is finite there exist an $n$ and an integer vector $\boldsymbol{a}$ such that $\Lambda \subset A_{n}+\boldsymbol{a}$. This proves that $\limsup A_{0}(\Lambda) \leqq A_{0}$. For any $n$ and sufficiently large $\Lambda$ there exists an integer vector $\boldsymbol{a}$ such that $\Lambda_{n}+\boldsymbol{a} \subset \Lambda$. This proves that $\liminf A_{0}(\Lambda) \geqq A_{0}$.

Definition 8.8. $N(A, r)$ is defined for $r>0$ as the number of zeros of $P(\Lambda ; x)$ with modulus less than $r$. For $r \leqq 0$, one defines $N(\Lambda, r)=0$.

Definition 8.9. $n^{*}(\Lambda, r) \equiv N(\Lambda, r) / N(\Lambda), n^{*}(n, r) \equiv n^{*}\left(\Lambda_{n}, r\right)$. 
Lemma 8.3. The following limit exists for all $r$ :

$$
\lim _{n \rightarrow \infty} n^{*}(n, r)=n^{*}(r) .
$$

The convergence is uniform in $r$; indeed for all $r$ :

$$
\left|n^{*}(n, r)-n^{*}(r)\right| \leqq 4 v 2^{-n} .
$$

Proof. One has (with $N_{1}=N\left(\Lambda_{1}\right)$ ):

$$
N\left(\Lambda_{n}\right)=N_{1}\left(2^{n}-1\right)^{v} .
$$

If one deletes the middle rows of unit cells in $\Lambda_{n}$, i.e. the vertices with $2^{n-1}-1 \leqq x_{j}<2^{n-1}$ for at least one of its coordinates, then $\Lambda_{n}$ is divided into $2^{v}$ disjoint cubes $A_{n-1}$. From the interlacing statement of Theorem 4.1 one then gets

$$
\left|N\left(\Lambda_{n}, r\right)-2^{v} N\left(\Lambda_{n-1}, r\right)\right| \leqq N\left(\Lambda_{n}\right)-2^{v} N\left(\Lambda_{n-1}\right),
$$

because the right side of (8.9) is the number of deleted vertices; as each vertex is deleted $N(\Lambda, r)$ either increases or decreases by exactly one. Since

$$
\left|1-2^{v} N\left(\Lambda_{n-1}\right) / N\left(\Lambda_{n}\right)\right| \leqq\left|1-\left(1-2^{1-n}\right)^{v}\right| \leqq 2 v 2^{-n},
$$

one gets from Eq. (8.9):

$$
\left|n^{*}(n, r)-n^{*}(n-1, r)\right| \leqq 4 v 2^{-n} .
$$

Eq. (8.7), and thereby the lemma, is an immediate consequence of Eq. (8.11).

Theorem 8.4. If $\{\Lambda\}$ is a sequence of section graphs which tends to infinity, then the following limit is uniform in $r$

$$
\lim n^{*}(\Lambda, r)=n^{*}(r) .
$$

Proof. Take $\boldsymbol{a}=\left\{2^{n}+1,2^{n}+1, \ldots, 2^{n}+1\right\}$ and pack $\Lambda$ with $N_{\boldsymbol{a}}^{-}(\Lambda)$ copies of the cube $\Lambda(\boldsymbol{a})$. Next, delete the unit cells at the boundaries of the cubes $\Lambda(\boldsymbol{a})$. As above, one then gets

$$
\left|N(\Lambda, r)-N_{\boldsymbol{a}}^{-}(\Lambda) N\left(\Lambda_{n}, r\right)\right| \leqq N(\Lambda)-N_{\boldsymbol{a}}^{-}(\Lambda) N\left(\Lambda_{n}\right) .
$$

Further, one has:

$$
\begin{aligned}
0 & \leqq 1-N_{\boldsymbol{a}}^{-}(\Lambda) N\left(\Lambda_{n}\right) / N(\Lambda) \\
& \leqq 1-\left[N_{\boldsymbol{a}}^{-}(\Lambda) / N_{\boldsymbol{a}}^{+}(\Lambda)\right]\left[N\left(\Lambda_{n}\right) / N(\Lambda(\boldsymbol{a}))\right] \\
& \leqq 1-\left[N_{\boldsymbol{a}}^{-}(\Lambda) / N_{\boldsymbol{a}}^{+}(\Lambda)\right]\left[1-2 v 2^{-n}\right] .
\end{aligned}
$$

If $N_{0}$ is chosen such that

$$
1-N_{\boldsymbol{a}}^{-}(\Lambda) / N_{\boldsymbol{a}}^{+}(\Lambda)<2^{-n+1}
$$


when $N(\Lambda)>N_{0}$, then one has for $N(\Lambda)>N_{0}$

$$
\left|n^{*}(\Lambda, r)-n^{*}(n, r)\right| \leqq 4(v+1) 2^{-n}
$$

Using Eq. (8.7),

$$
\left|n^{*}(\Lambda, r)-n^{*}(r)\right| \leqq 8(v+1) 2^{-n}
$$

which proves the theorem.

Remark 8.5. If $\phi(r)$ and $\psi(r)$ are two non-decreasing functions which satisfy

$$
\begin{gathered}
\phi(r)=\psi(r), \text { for } r \leqq a \text { and } r \geqq b, \\
|\phi(r)-\psi(r)|<\varepsilon, \text { for } a \leqq r \leqq b,
\end{gathered}
$$

and if $f(r)$ is continuous and non-decreasing in the closed interval $[a, b]$ and differentiable in the open interval $(a, b)$ then

$$
\left|\int_{-\infty}^{\infty} f(r) d \phi(r)-f(r) d \psi(r)\right|<\varepsilon[f(b)-f(a)] .
$$

If one defines a function

$$
g(\Lambda)=\int_{-\infty}^{\infty} \gamma(r) d n^{*}(\Lambda, r)
$$

for $\gamma$ continuous, then, as $N(\Lambda) \rightarrow \infty$,

$$
\lim _{\Lambda \rightarrow \infty} g(\Lambda)=\int_{-\infty}^{\infty} \gamma(r) d n^{*}(r)=g
$$

If $\gamma(r)$ is differentiable and non-decreasing in $0 \leqq r \leqq A_{0}$ and if $\left\{n^{*}(r)\right.$ $-n^{*}(\Lambda, r) \mid<\varepsilon$, then

$$
|g(\Lambda)-g|<\varepsilon\left[\gamma\left(A_{0}\right)-\gamma(0)\right]
$$

This is a useful tool for proving uniformity of convergence.

Definition 8.10.

$$
g(\Lambda ; \mu) \equiv \frac{1}{2} \mu+N(\Lambda)^{-1} \ln P\left(\Lambda ; e^{-\frac{1}{2} \mu}\right) .
$$

Lemma 8.5. $g(\Lambda, \mu)=\frac{1}{2} \mu+\frac{1}{2} \int_{-\infty}^{\infty} \ln \left(e^{-\mu}+r^{2}\right) d n^{*}(\Lambda, r)$.

Proof. Trivial.

Definition 8.11.

$$
g(\mu) \equiv \frac{1}{2} \mu+\frac{1}{2} \int \ln \left(e^{-\mu}+r^{2}\right) d n^{*}(r) .
$$


Theorem 8.6. If $\Lambda$ tends to infinity, then, for all $\mu$,

$$
\lim _{\Lambda \rightarrow \infty} g(\Lambda ; \mu)=g(\mu) .
$$

The convergence is uniform on subsets of the form $\mu \leqq \mu_{0}$.

Proof. From Remark 8.5 it follows that

$$
|g(\Lambda ; \mu)-g(\mu)|<\varepsilon \ln \left(1+A_{0}^{2} e^{\mu}\right),
$$

when $N(\Lambda)>N_{0}$, where $N_{0}$ is chosen to make

$$
\left|n^{*}(\Lambda, r)-n^{*}(r)\right|<\varepsilon
$$

for $N(\Lambda)>N_{0}$.

Remark 8.6. One can alternatively prove the existence of the grand canonical limit $g(\mu)$ directly, and then infer the existence of the distribution $n^{*}(r)$ from the power series expansion of $g(\mu)$ in $e^{\mu}$. This follows by the theory of the Hamburger moment problem from the fact that the domain of increase of $n^{*}(r)$ is finite by Theorem 8.4.

Definition 8.12. If, for a given $\Lambda, d_{0}$ is the maximum value of $d$ for which $Z_{d}$ is strictly positive, we define

$$
\varrho_{0}(\Lambda)=d_{0} / N(\Lambda) .
$$

Lemma 8.7. $\quad \varrho_{0}(\Lambda)=\lim _{\mu \rightarrow \infty} \frac{d g(\Lambda, \mu)}{d \mu}$,

$$
\lim _{\Lambda \rightarrow \infty} \varrho_{0}(\Lambda)=\lim _{\mu \rightarrow \infty} \frac{d g(\mu)}{d \mu} \equiv \varrho_{0} .
$$

Proof. Eq. (8.28) is a trivial consequence of the fact that $\exp [g(\Lambda ; \mu)]$ is a polynomial in $\exp \left(\frac{1}{2} \mu\right)$; the non-trivial part of the theorem is $(8.29)$, for it says that we can interchange the limits $\mu \rightarrow \infty$ and $\Lambda \rightarrow \infty$. The left side of (8.29) is $\lim _{\Lambda \rightarrow \infty} \lim _{\mu \rightarrow \infty} \int r^{2}\left(e^{-\mu}+r^{2}\right)^{-1} d n^{*}(\Lambda, r)$ and the right side is the same with the limits reversed. On the right side we can take the limit $\Lambda \rightarrow \infty$ which, by Remark 8.5, means that $n^{*}(\Lambda, r) \rightarrow n^{*}(r)$. Now it is clear that for any increasing $m(r)$, with $m(\infty)=1$ and with $m(0)=0$ for $r<0$,

$$
\lim _{\mu \rightarrow \infty} \int r^{2}\left(e^{-\mu}+r^{2}\right)^{-1} d m(r)=1-m(0+),
$$

where $m(0+)=\lim _{r \rightarrow 0} m(r)$. Thus, to prove the theorem we require that $\lim _{\Lambda \rightarrow \infty} n^{*}(\Lambda, 0+)=n^{*}(0+)$. This, in turn, is a consequence of the uniform convergence of $n^{*}(\Lambda, \cdot)$ to $n^{*}(\cdot)$.

Definition 8.13. For $0 \leqq \varrho \leqq \varrho_{0}(\Lambda)$ we define the function $h(\Lambda, \varrho)$, for $\varrho N(\Lambda)$ integral, by

$$
h(\Lambda, \varrho)=N(\Lambda)^{-1} \ln Z_{\varrho N(\Lambda)} .
$$


For other values of $\varrho$ we define $h(\Lambda, \varrho)$ by linear interpolation between $\varrho_{1}$ and $\varrho_{2}$ which are chosen such that $\varrho_{1}<\varrho<\varrho_{2}, N \varrho_{1}$ is integral and $N\left(\varrho_{2}-\varrho_{1}\right)=1$. For $\varrho>\varrho_{0}$ we define $h(\Lambda, \varrho)=-\infty$. It is a very important consequence of Newton's inequality (Theorem 7.1) that $h(\Lambda, \varrho)$ is concave in $\varrho$ for $0 \leqq \varrho \leqq 1$.

Definition 8.14. For $0 \leqq \varrho \leqq 1$

$$
h(\varrho) \equiv \inf \{-\varrho \mu+g(\mu):-\infty<\mu<\infty\} .
$$

Theorem 8.8A. $\limsup _{\Lambda \rightarrow \infty} h(\Lambda, \varrho)=h(\varrho), \quad 0 \leqq \varrho \leqq 1$.

Proof.

$$
\begin{aligned}
g(\Lambda, \mu) & \geqq \max \left\{\mu N(\Lambda)^{-1} d+N(\Lambda)^{-1} \ln Z_{d}: d \leqq N(\Lambda)\right\} \\
& =\sup \{\varrho \mu+h(\Lambda, \varrho): 0 \leqq \varrho \leqq 1\} .
\end{aligned}
$$

Choose $N_{0}$ such that $|g(\Lambda, \mu)-g(\mu)|<\varepsilon$ for $N(\Lambda)>N_{0}$ and for $\mu \leqq \mu_{0}<\infty$. Then

$$
g(\mu)+\varepsilon \geqq \sup \{\varrho \mu+h(\Lambda, \varrho): 0 \leqq \varrho \leqq 1\},
$$

i.e. for all $\varrho$ and $N(\Lambda)>N_{0}$ and $\mu \leqq \mu_{0}$

Therefore,

$$
h(\Lambda, \varrho) \leqq g(\mu)-\varrho \mu+\varepsilon .
$$

$$
\limsup _{\Lambda \rightarrow \infty} h(\Lambda, \varrho) \leqq \inf \left\{g(\mu)-\varrho \mu: \mu \leqq \mu_{0}\right\}
$$

for all $\mu_{0}$, and (8.33) follows.

Theorem 8.8B. For $0 \leqq \varrho<\varrho_{0}$,

$$
\liminf _{\Lambda \rightarrow \infty} h(\Lambda, \varrho)=h(\varrho) .
$$

Proof. One has

$$
\begin{aligned}
g(\Lambda, \mu) & \leqq N(\Lambda)^{-1} \ln N(\Lambda)+\max \left\{\mu N(\Lambda)^{-1} d+N(\Lambda)^{-1} \ln Z_{d}: d \leqq N(\Lambda)\right\} \\
& =N(\Lambda)^{-1} \ln N(\Lambda)+\sup \{\varrho \mu+h(\Lambda, \varrho) ; 0 \leqq \varrho \leqq 1\} .
\end{aligned}
$$

Since $h(\Lambda, \varrho)$ is concave in $\varrho$,

$$
h(\Lambda, \varrho)=\inf \{-\varrho \mu+g(\Lambda, \mu):-\infty<\mu<\infty\}-N(\Lambda)^{-1} \ln N(\Lambda) \text {. }
$$

Now choose $\mu_{0}$ such that

$$
d g\left(\mu_{0}\right) / d \mu_{0}=\frac{1}{2}\left(\varrho_{0}+\varrho\right)
$$

and then choose $N_{0}$ such that for $N(\Lambda)>N_{0}$

$$
\begin{gathered}
N(\Lambda)^{-1} \ln N(\Lambda)<\frac{1}{2} \varepsilon, \\
\partial g\left(\Lambda, \mu_{0}\right) / \partial \mu_{0}>\varrho,
\end{gathered}
$$


and for $\mu \leqq \mu_{0}$

$$
g(\Lambda, \mu)-g(\mu)>-\frac{1}{2} \varepsilon .
$$

From (8.41) it follows that for $N(\Lambda)>N_{0}$

$$
\inf \{-\varrho \mu+g(\Lambda, \mu): \infty<\mu<\infty\}=\inf \left\{-\varrho \mu+g(\Lambda, \mu):-\infty<\mu \leqq \mu_{0}\right\} .
$$

From (8.42) it follows that for $N(A)>\mathrm{N}_{0}$

$$
\inf \left\{-\varrho \mu+g(\Lambda, \mu):-\infty<\mu \leqq \mu_{0}\right\} \geqq h(\varrho)-\frac{1}{2} \varepsilon .
$$

Consequently, for $N(\Lambda)>N_{0}$

$$
h(\Lambda, \varrho) \geqq h(\varrho)-\varepsilon,
$$

which proves the theorem.

Remark 8.7. The limit

$$
\lim _{\Lambda \rightarrow \infty} h\left(\Lambda, \varrho_{0}\right)
$$

will generally depend on the specific boundary condition and does not need to exist. Hammersley [59] proved that for simple cubic lattices in any dimension and for $\{\Lambda\}$ being rectangular boxes with faces perpendicular to the directions of the edges and for an even number of vertices one has

$$
\lim _{\Lambda \rightarrow \infty} h\left(\Lambda, \varrho_{0}\right)=h\left(\varrho_{0}\right) .
$$

We conjecture that (8.44) holds generally for cyclic boundary conditions provided $\Lambda$ is chosen to have a dimer arrangement with the density $\varrho_{0}$.

\section{Thermodynamic Limit for Monomer-Dimer Systems: Analyticity Properties}

In this section we shall combine the results of Sections IV and VIII in order to furnish the general analyticity properties of the thermodynamic functions. We shall also prove the existence and analyticity of the infinite lattice correlation functions. We begin by restating the general analyticity condition of Theorem 4.9.

Definition 9.1. Let $G$ be a graph with general, complex edge weights. Write the edge weights as

$$
W(i, j)=-U(i, j)^{2}
$$

and assume

$$
U(i, j) \in D_{ \pm}(V(i, j), \theta), \quad \text { all } \quad i, j \in G,
$$


for some set of real, non-negative numbers $\{V(i, j)\}$ and some value of $\theta, 0 \leqq \theta \leqq \pi / 2$. Finally let $\bar{G}$ be the graph with the edge weights changed to $V(i, j)^{2}$. If $x$ is the monomer fugacity, we then say that $x \in \mathscr{A}(G)$ if $\theta$ and the set of numbers $\{V(i, j)\}$ can be chosen such that (9.2) is fulfilled and simultaneously

$$
x \in D_{ \pm}^{C}\left(A_{0}(\bar{G}), \theta\right)=D_{+}^{C}\left(A_{0}(\bar{G}), \theta\right) \cup D_{-}^{C}\left(A_{0}(\bar{G}), \theta\right) .
$$

If (9.3) is changed to

$$
x \in D_{ \pm}^{C}\left(A_{0}(\bar{G})+\varepsilon, \theta\right)
$$

then $x \in \mathscr{A}_{\varepsilon}(G)$.

Finally let $L$ be a lattice (Definition 8.1) with complex edge weights, let the edge weights be written in the form (9.1) and satisfy (9.2) with a set of weights $\{V(i, j)\}$ such that $\bar{L}$ (defined analogously to $\bar{G}$ ) is a lattice (i.e. that the translation invariance is preserved), and let $A_{0}$ be taken to be the value of $A_{0}$ obtained for $\bar{L}$ by the limit in Theorem 8.2. If $\theta$ and the set of numbers $\{V(i, j)\}$ can be chosen such that both (9.2) is satisfied and simultaneously

$$
x \in D_{ \pm}^{C}\left(A_{0}, \theta\right),
$$

then we say that $x \in \mathscr{A}(L)$.

Next, we modify the definition of the free energy slightly from Definition 8.10.

Definition 9.2.

$$
g_{m}(G ; x) \equiv N(G)^{-1} \ln P(G ; x)-\ln x
$$

where the branch cuts of the right side of $(9.6)$ are the $N(G)$ line segments $(z, 0)$, where $z$ is any one of the zeros of $P(G ; x)$.

One then has the following lemma as a trivial corollary of Theorem 4.9.

Lemma 9.1. If $G$ is a weighted graph and if $x \in \mathscr{A}_{\varepsilon}(G)$ then

$$
\left|g_{m}(G ; x)\right|<|\ln \varepsilon|+2 \pi .
$$

By application of Vitali's theorem one easily proves the following theorem from Lemma 9.1 and Theorem 8.6:

Theorem 9.2. Let $L$ be a lattice with complex edge weights and let $\{\Lambda\}$ be a sequence of section graphs that tends to infinity in the sense of Van Hove. Then the limit

$$
\lim _{\Lambda \rightarrow \infty} g_{m}(\Lambda ; x)=g_{m}(x)
$$

exists, is independent of the sequence, and is uniform on closed subsets of the following domains:

(i) If the edge weights are held fixed then $g_{m}(x)$ is analytic in $x \in \mathscr{A}(L)$. 
(ii) If $g_{m}(x)$ is also considered as a function of the edge weights then it is analytic on the following domain:

$$
\begin{array}{r}
\bigcup_{\{V(i, j)\}} \bigcup_{0 \leqq \theta \leqq \pi / 2}\left\{(x,\{W\}): x \in D_{ \pm}^{C}\left(A_{0}, \theta\right), W(i, j)=-U(i, j)^{2}\right. \\
\text { and } \left.U(i, j) \in D_{ \pm}(V(i, j), \theta)\right\},
\end{array}
$$

where the allowed $\{V(i, j)\}$ are translation invariant.

Remark. If all the edge weights are real and positive, then Theorem 9.2 states that the free energy is analytic in $x$ in the cut $x$-plane where the cut runs from $-i A_{0}$ to $i A_{0}$ along the imaginary axis. One can then obtain a natural expansion variable $s$ by the substitution

$$
\begin{aligned}
& x=\frac{1}{2} A_{0}\left(1-s^{2}\right) / s \\
& \left.s=\left(x / A_{0}\right)\left[1+A_{0}^{2} / x^{2}\right)^{\frac{1}{2}}-1\right]
\end{aligned}
$$

which maps the cut $x$-plane conformally onto the unit disk of the $s$-plane. A power series in $s$ will then converge in the whole physical region.

As mentioned in the introduction, the variable $s$ is essentially the same as the expansion variable, $y_{2}$, used by Nagle [27]. To establish the connection we note that if $A_{0}$ is replaced by some $A^{\prime}>A_{0}$ in $(9.10,11)$, one obtains an expansion variable $s^{\prime}$ with the same property that the analyticity region is mapped into the unit $s^{\prime}$-disk. Naturally, the best $A^{\prime}$ to use is $A_{0}$, but in the absence of accurate knowledge of $A_{0}$ we can use the bound given by Theorem 4.3. For a connected lattice, all of whose non-zero edge weights are a constant, $W$, and which has coordination number, $q$, the upper bound is

$$
A^{\prime}=[4 W(q-1)]^{\frac{1}{2}} \text {. }
$$

It is this $A^{\prime}$ that appears in Nagle's $y_{2}$. We also note in passing that our previous report [61] contained an inferior estimate in which $(q-1)$ was replaced by $q$.

As the last point we turn to the correlation functions.

Definition 9.3. The correlation function $\varrho(G, S)$ is defined for subsets $S$ of the graph $G(S \subset G)$ by

$$
\varrho(G, S ; x)=x^{N(S)} P(G-S ; x) / P(G ; x) .
$$

We first prove two lemmas which are the equivalent of Lemma 9.1 and Theorem 8.2 and then obtain the final theorem by an application of Vitali's theorem. For the sake of the proof of the lemmas it is more convenient to work with the function

$$
f\left(G, S ; x_{1}, \ldots, x_{N}\right)=P\left(G-S ; x_{1}, \ldots, x_{N}\right) / P\left(G ; x_{1}, \ldots, x_{N}\right) .
$$


Lemma 9.3. If $G$ is a weighted graph and if $x \in \mathscr{A}_{\varepsilon}(G)$ then

$$
|f(G, S ; x, \ldots, x)| \leqq \varepsilon^{-N(S)} .
$$

Proof. If we choose

$$
x_{j}= \begin{cases}x^{\prime} & j \in S \\ x^{\prime \prime} & j \in G-S\end{cases}
$$

then, in an obvious notation, we have

$$
P\left(G ; x^{\prime}, x^{\prime \prime}\right)=P\left(G-S ; x^{\prime \prime}\right) \prod_{j=1}^{N(S)}\left[x^{\prime}-a_{j}\left(x^{\prime \prime}\right)\right]
$$

since the coefficient of $\left(x^{\prime}\right)^{N(S)}$ is $P\left(G-S ; x^{\prime \prime}\right)$. Now $P\left(G-S ; x^{\prime \prime}\right)$ is surely not zero if $x^{\prime \prime} \in \mathscr{A}(G)$ and therefore

$$
f\left(G, S ; x^{\prime}, x^{\prime \prime}\right)=\prod_{j=1}^{N(S)}\left[x^{\prime}-a_{j}\left(x^{\prime \prime}\right)\right]^{-1} .
$$

It follows from Theorem 4.9 that if $x^{\prime \prime} \in D_{+}^{C}\left(A_{0}(\bar{G}), \theta\right)$ then

$$
a_{j}\left(x^{\prime \prime}\right) \in D_{+}\left(A_{0}(\bar{G}), \theta\right) .
$$

Consequently, if $x=x^{\prime}=x^{\prime \prime} \in \mathscr{A}_{\varepsilon}(G)$ then

$$
\left|x^{\prime}-a_{j}\left(x^{\prime \prime}\right)\right|>\varepsilon,
$$

which proves the lemma.

Lemma 9.4. If $G$ is a weighted graph whose edge weights are real and positive, $N(S)=N$, and if $x$ is purely imaginary and

$$
-i x>A_{0}(G)
$$

then $i^{N(S)} f\left(G, S ; m_{1} x, \ldots, m_{N} x\right)$ is monotone decreasing in each of the variables, $m_{j}(j \in G-S)$, when $m_{k}$ is real and greater than one for $k=1, \ldots, N$.

Proof. Suppose $\{1\} \subset G-S$. Then if $\hat{G}=G-1$ $\partial f\left(G, S ; m_{1} x, \ldots, m_{N} x\right) / \partial m_{1}$

$$
\begin{aligned}
= & x\left[P\left(G ; m_{1} x, \ldots, m_{N} x\right) P\left(\hat{G}-S ; m_{2} x, \ldots, m_{N} x\right)\right. \\
& -P\left(\hat{G} ; m_{2} x, \ldots, m_{N} x\right) \\
& \left.\cdot P\left(G-S ; m_{1} x, \ldots, m_{N} x\right)\right] / P^{2}\left(G ; m_{1} x, \ldots, m_{N} x\right) .
\end{aligned}
$$

Application of Eqs. (2.7), (2.10) and (2.11) then yields

$$
\begin{aligned}
& \left(\prod_{j \in S} m_{j}\right) i^{N(S)} \partial f\left(G, S ; m_{1} x, \ldots, m_{N} x\right) / \partial m_{1} \\
& =y m_{1}^{-1}\left[Q\left(G^{\prime} ; y\right) Q\left((\hat{G}-S)^{\prime} ; y\right)-Q\left(G^{\prime} ; y\right) Q\left((G-S)^{\prime} ; y\right)\right] / Q^{2}\left(G^{\prime} ; y\right),
\end{aligned}
$$

with $y=-i x$. The monotonicity then follows from Theorem 6.3 since $y>A_{0}(G) \geqq A_{0}\left(G^{\prime}\right)$ by assumption. 
Theorem 9.5. Let $S \subset G_{1} \subset G_{2} \subset \cdots$ be an infinite sequence of section graphs with complex edge weights. Then the limit

$$
\lim _{i \rightarrow \infty} \varrho\left(G_{i}, S ; x\right)=\varrho(S ; x)
$$

exists uniformly on closed subsets of the following domains

(i) If the edge weights are held fixed then $\varrho(S ; x)$ is analytic on $\operatorname{Int}\left[\bigcap_{i} \mathscr{A}\left(G_{i}\right)\right]$.

(ii) If $\varrho(S ; x)$ is also considered as a function of any finite number of distinct edge weights then it is analytic on

$$
\begin{aligned}
\operatorname{Int}\left[\bigcap _ { i } \left(\bigcup _ { \{ V ( i , j ) \} } \bigcup _ { 0 \leqq \theta \leqq \pi / 2 } \left\{(x,\{W\}): x \in D_{ \pm}^{C}\left(A_{0}\left(G_{i}\right), \theta\right),\right.\right.\right. \\
\left.\left.W(i, j)=-U(i, j)^{2} \text { and } U(i, j) \in D_{ \pm}(V(i, j), \theta)\right\}\right] .
\end{aligned}
$$

If the sequence is a sequence of section graphs of a lattice, $L$, and if the $\{V(i, j)\}$ in (9.25) are restricted to be translation invariant, then the limit is independent of the sequence when the sequence tends to infinity in the sense of Van Hove, provided the distance of $S$ from the boundary of $G_{i}$ tends to infinity as $i \rightarrow \infty$.

Proof. We notice that $G_{i}$ can be thought of as $G_{i+1}$ in which the vertex weights on the vertices belonging to $G_{i+1}-G_{i}$ are infinite. Consequently, if the edge weights are positive and if $x$ is sufficiently large and imaginary, Lemmas 9.3 and 9.4 tell us that $\left\{\varrho\left(G_{i}, S ; x\right)\right\}$ is a monotone bounded sequence and hence has a limit. The rest follows from Vitali's theorem. The uniqueness of the limit in the case of a Van Hove sequence follows from the same cube packing arguments as in Section VIII.

\section{References}

1. Roberts, J. K.: Proc. Roy. Soc. (London) A 152, 469 (1935).

2. - Proc. Roy. Soc. (London) A 161, 141 (1937).

3. - Proc. Cambridge Phil. Soc. 34, 399 (1938).

4. - Miller, A. R.: Proc. Cambridge Phil. Soc. 35, 293 (1939).

5. Readhead, P. A.: Trans. Faraday Soc. 57, 641 (1961).

6. Rossington, D. R., Bost, R.: Surface Sci. 3, 202 (1965).

7. Lichtman, D., McQuistan, R. B. : J. Math. Phys. 8, 2441 (1967).

8. McQuistan, R. B., Lichtman, D. : J. Math. Phys. 9, 1660 (1968).

9. - J. Math. Phys. 10, 2205 (1969).

10. - Lichtman, S. J.: J. Math. Phys. 11, 3095 (1970).

11. Fowler, R. H., Rushbrooke, G. S.: Trans. Faraday Soc. 33, 1272 (1937).

12. Guggenheim, A.: Trans. Faraday Soc. 33, 151 (1937).

13. Chang, T. S.: Proc. Roy. Soc. (London) A 169, 512 (1939).

14. - Proc. Cambridge Phil. Soc. 35, 265 (1939).

15. Miller, A. R.: Proc. Cambridge Phil. Soc. 38, 109 (1942). 
16. Orr, W.J.C.: Trans. Faraday Soc. 40, 306 (1944).

17. McGlashan, M.L.: Trans. Faraday Soc. 47, 1042 (1951).

18. Miller, A. R.: Proc. Cambridge Phil. Soc. 39, 54 (1943).

19. - Proc. Cambridge Phil. Soc. 39, 131 (1943).

20. Orr, W.J.C.: Trans. Faraday Soc. 40, 320 (1944).

21. Guggenheim, E. A.: Proc. Roy. Soc. (London) A 183, 203 (1944).

22. - Proc. Roy. Soc. (London) A 183, 213 (1944).

23. We shall not attempt to give a complete bibliography of the Flory-Huggins theory; the reader is referred to standard textbooks. The earliest references seem to be P.J. Flory: J. Chem. Phys. 10, 51 (1942) and Huggins: Ann. N. Y. Acad. Sci. 43, 9 (1942).

24. Guggenheim, E. A.: Mixtures, Chapter X. Oxford: Claredon Press 1952.

25. Rushbrooke, G. S., Scoins, H. I., Wakefield, A. J. : Discussions Farad. Soc. 15, 57 (1953).

26. Travena, D. H.: Proc. Phys. Soc. 84, 969 (1964).

27. Nagle, J. F.: Phys. Rev. 152, 190 (1966).

28. Gaunt, D. S.: Phys. Rev. 179, 174 (1969).

29. Bellemans, A., Fuks, S.: Physica 50, 348 (1970).

30. Runnels, L. K.: J. Math. Phys. 11, 842 (1970).

31. Baxter, R. J.: J. Math. Phys. 9, 650 (1968).

32. Craen, J.van, Bellemans, A.: Bull. Acad. Pol. Sci. 19, 45 (1971).

33. Hammersley, J. M.: In: Proceedings of the 2 nd Annual Conference on Computational Physics, pp. 1-8 (Institute of Physics and Physical Society, London (1970)).

34. Baxendale, J. H., Enüstün, B. V., Stern, J.: Phil. Trans. Roy. Soc. (London) A 243, 169 (1951).

35. Everett, D. H., Penney, M. F.: Proc. Roy. Soc. (London) A 212, 164 (1952).

36. Tompa, H.: J. Chem. Phys. 16, 292 (1948).

37. Brøndsted, J. N., Koefoed, J.: Kgl. Danske Videnskab. Selskob. Mat-Fys. Medd. 22, No. 17 (1946).

38. Tompa, H.: Trans. Faraday Soc. 45, 101 (1949).

39. Pizzini, S., Morlotti, R., Wagner, V.: J. Electrochem. Soc. 116, 915 (1969).

40. Cohen,E. G. D., De Boer, J., Salsburg, Z. W.: Physica 21, 137 (1955).

41. Conway, B. E., Verall, R. E.: J. Phys. Chem. 70, 1473 (1966).

42. Fisher, M.E., Temperley, H. N. V.: Rev. Mod. Phys. 32, 1029 (1960).

43. Katsura, S., Inawashiro, S.: Rev. Mod. Phys. 32, 1031 (1960).

44. Kasteleyn, P. W.: Physica, Grav. 27, 1209 (1961).

45. Temperley,H.N.V., Fisher, M.E.: Phil. Mag. Serie 8 6, 1061 (1961).

46. Fisher, M.E.: Phys. Rev. 124, 1664 (1961).

47. Kasteleyn, P. W.: J. Math. Phys. 4, 287 (1963).

48. Montroll,E.W.: In: Applied combinatorial mathematics (Ed. F. Beckenbach). New York: J. Wiley \& Sons, 1964.

49. Lieb, E. H.: J. Math. Phys. 8, 2339 (1967).

50. Gibberd, R. W.: Can. J. Phys. 46, 1681 (1968).

51. Wu, T. T.: J. Math. Phys. 3, 1265 (1962).

52. Ferdinand, A.E.: J. Math. Phys. 8, 2332 (1967).

53. Hammersley, J. M., Feuerverger, A., Izenman, A., Mahani, S.: J. Math. Phys. 10, 443 (1969).

54. Fisher, M.E., Stephenson, J.: Phys. Rev. 132, 1411 (1963).

55. Hartwig, R. E.: J. Math. Phys. 7, 286 (1966).

56. Bondy, J. A., Welsh, D. J. A.: Proc. Cambridge Phil. Soc. Math. Phys. Sci. 62, 503 (1966).

57. Hammersley, J. M.: Proc. Cambridge Phil. Soc. Math. Phys. Sci. 64, 455 (1968).

58. - Menon, V. V.: J. Inst. Math. Appl. 6, 341 (1970).

59. - In: Research papers in statistics. Festschrift für J. Neyman, p. 125 (Editor, F. N. David). New York: John Wiley \& Sons 1966. 
60. Heilmann, O.J.: Existence of phase transitions in certain lattice gases with repulsive potentials (to be published).

61. - Lieb, E. H.: Phys. Rev. Letters 24, 1412 (1970).

62. Kunz, H.: Phys. Letters 32 A, 311 (1970).

63. Gruber. C.. Kunz, H.: Commun. math. Phys. 22, 133 (1971).

64. Dobrushin, R. L.: Funct. Anal. Appl. 2, No. 4, 44 (1968), (English translation 2, 302 (1968)).

65. Essam, J. W.. Fisher, M.E.: Rev. Mod. Phys. 42. 271 (1970).

66. Szegö, G.: Orthogonal polynomials (American Mathematical Society, Colloquium Publications Vol. XXIII, Providence 1939).

67. Ruelle, D.: Statistical mechanics. New York: W. A. Benjamin 1969.

68. Fisher, M.E.: J. Math. Phys. 7, 1776 (1966).

69. Lee, T. D., Yang, C. N.: Phys. Rev. 87, 410 (1952).

70. Asano, T.: J. Phys. Soc. Japan 29, 350 (1970); Phys. Rev. Letters 24, 1409 (1970).

71. Suzuki, M., Fisher, M. E.: J. Math. Phys. 12, 235 (1971).

72. Ginibre, J.: Phys. Letters 24 A, 223 (1967).

E. H. Lieb

Department of Mathematics, 2--375

Massachusetts Institute of Technology

Cambridge. Mass. 02139, USA 